\title{
A class of hemivariational inequalities for nonstationary Navier-Stokes equations ${ }^{1}$
}

\author{
Changjie Fang ${ }^{2}$, Weimin $\mathrm{Han}^{3}$, Stanisław Migórski ${ }^{4}$, Mircea Sofonea $^{5}$
}

\begin{abstract}
This paper is devoted to the study of a class of hemivariational inequalities for the time-dependent Navier-Stokes equations, including both boundary hemivariational inequalities and domain hemivariational inequalities. The hemivariational inequalities are analyzed in the framework of an abstract hemivariational inequality. Solution existence for the abstract hemivariational inequality is explored through a limiting procedure for a temporally semi-discrete scheme based on the backward Euler difference of the time derivative, known as the Rothe method. It is shown that solutions of the Rothe scheme exist, they contain a weakly convergent subsequence as the time step-size approaches zero, and any weak limit of the solution sequence is a solution of the abstract hemivariational inequality. It is further shown that under certain conditions, a solution of the abstract hemivariational inequality is unique and the solution of the abstract hemivariational inequality depends continuously on the problem data. The results on the abstract hemivariational inequality are applied to hemivariational inequalities associated with the time-dependent Navier-Stokes equations.
\end{abstract}

Key words. Navier-Stokes equations, hemivariational inequality, Rothe method, existence, uniqueness, continuous dependence

AMS subject classifications. 76D05, 47J20, 35K85, 34G25, 74H20, 74H25

\footnotetext{
${ }^{1}$ The work was partially supported by supported by the Marie Curie International Research Staff Exchange Scheme Fellowship within the 7th European Community Framework Programme under Grant Agreement No. 295118, the National Science Center of Poland under the Maestro Advanced Project no. DEC2012/06/A/ST1/00262. The first author was also supported by the National Natural Science Foundation of China (No. 11426055) and by Basic and Advanced Research Project of CQ CSTC (No. cstc2014jcyjA00044). The second author was also supported by NSF under grant DMS-1521684 and the Simons Foundation. The third author was also supported by the National Science Center of Poland under Grant no. N N201 604640 and the International Project co-financed by the Ministry of Science and Higher Education of Republic of Poland under Grant no. W111/7.PR/2012.

${ }^{2}$ College of Science, Chongqing University of Posts and Telecommunications, Chongqing 400065, China; e-mail: fangcj@cqupt.edu.cn

${ }^{3}$ Department of Mathematics, University of Iowa, Iowa City, IA 52242-1410, USA; School of Mathematics and Statistics, Xi'an Jiaotong University, Xi'an, Shaanxi 710049, China; e-mail: weimin-han@uiowa.edu

${ }^{4}$ Faculty of Mathematics and Computer Science, Jagiellonian University, Institute of Computer Science, ul. Stanisława Łojasiewicza 6, 30348 Krakow, Poland; e-mail: Migorski@ii.uj.edu.pl

${ }^{5}$ Laboratoire de Mathématiques et Physique, Université de Perpignan Via Domitia, 52 Avenue Paul Alduy, 66860 Perpignan, France. E-mail: sofonea@univ-perp.fr
} 


\section{Introduction}

Variational inequalities and hemivariational inequalities each form an important family of nonlinear problems with applications in several fields such as mechanics, physics, engineering, and economics. One of the bases for its development was the contribution of Fichera ([15]) on the solution of the frictionless contact problem between a linearly elastic body and a rigid foundation, posed by Signorini ([44]). Mathematical analysis of variational inequalities started in 1960s. The foundations of the mathematical theory of variational inequalities were laid in $[45,24,34,6]$. In particular, Stampacchia coined the term "Variational Inequality" in [45]. The study of variational inequalities and their applications was popularized by several early monographs, such as [14, 29, 3]. The monographs [19, 18, 26] provide comprehensive coverage of numerical methods and their analysis for solving various variational inequalities. Applications of variational inequalities in contact mechanics and plasticity can be found in [28, 23, 21].

The notion of hemivariational inequalities was first introduced by Panagiotopoulos in early 1980s ([41]) and is closely related to the development of the concept of the generalized gradient of a locally Lipschitz functional provided by Clarke $([9,10])$. Interest in hemivariational inequalities originated, similarly as in variational inequalities, in mechanical problems. From this point of view, the inequality problems in mechanics can be divided into two main classes: that of variational inequalities which is concerned with convex energy functionals (potentials), and that of hemivariational inequalities which is concerned with nonsmooth and nonconvex energy functionals (superpotentials). Through the formulation of hemivariational inequalities, problems involving nonmonotone, nonsmooth and multivalued constitutive laws, forces, and boundary conditions can be treated successfully, both theoretically and numerically. During the last three decades, hemivariational inequalities were shown to be very useful across a variety of subjects, and there is a large number of problems which lead to mathematical models expressed in terms of hemivariational inequalities. The mathematical literature dedicated to this field is growing rapidly. The theory, numerical solution and applications of hemivariational inequalities can be found in several monographs $([42,40,7,38])$ and the references therein. Analysis of the finite element method for solving hemivariational inequalities can be found in the monograph [25]. In the recent papers $[20,4]$, optimal order error estimates are derived, for the first time, for the linear finite element approximations of some hemivariational inequalities.

Time-dependent and time-independent Navier-Stokes equations have been research topics of substantial efforts in their mathematical theories, numerical solutions, computer simulations, and applications. In this regard, we refer the reader to $[46,47]$ for mathematical theories and to [17] for numerical analysis of initial-boundary or boundary value problems of the Navier-Stokes equations. Starting with the reference [16], variational inequalities for the Navier-Stokes equations or the Stokes equations are formulated and studied for viscous incompressible fluid flow problems involving leak or slip boundary conditions. Some recent references on the analysis and numerical solution of such variational inequalities include [13, 30, 31, 32, 33]. In the context of hemivariational inequalities associated with the Navier-Stokes equations, a stationary hemivariational inequality is studied in [35], and an evolutionary hemivariational inequality is studied in [36]; these two papers 
provide existence results to the hemivariational inequalities, as well as the solution uniqueness for the stationary hemivariational inequality. In this paper, we study hemivariational inequalities for the time-dependent Navier-Stokes equations, through a unified framework of an abstract problem. We explore the solution existence, uniqueness, and continuous dependence on the data for an abstract hemivariational inequality problem, and apply the results to the nonstationary hemivariational inequalities for the Navier-Stokes equations that are of the boundary type, corresponding to nonlinear slip boundary conditions, and of the domain type, corresponding to hydraulic flow controls.

The organization of the rest of the paper is as follows. In Section 2 we present some definitions and some auxiliary material. In Section 3, we introduce an abstract hemivariational inequality that includes as a particular case the hemivariational inequalities for the Navier-Stokes equations. The abstract hemivariational inequality is studied in Section 4 for the solution existence, and in Section 5 for the solution uniqueness and continuous dependence on data. The solution existence is proved through constructing a temporally semi-discrete approximation, known as the Rothe scheme, whose solutions converge to a solution of the abstract hemivariational inequality. Finally, in Section 6, we apply the results on the abstract hemivariational inequality to study the hemivariational inequalities for the nonstationary Navier-Stokes equations.

\section{Preliminaries}

All the function spaces in this paper are real. For a normed space $X$, we denote by $\|\cdot\|_{X}$ its norm, by $X^{*}$ its topological dual, and by $\langle\cdot, \cdot\rangle_{X^{*} \times X}$ the duality pairing between $X^{*}$ and $X$. The symbol $X_{w}$ is used for the space $X$ endowed with the weak topology. Weak convergence will be indicated by the symbol $\rightarrow$. The symbol $2^{X^{*}}$ represents the set of all subsets of $X^{*}$. For simplicity in exposition, in the following we always assume $X$ is a Banach space, unless stated otherwise.

We first recall the definition of generalized directional derivative and generalized gradient in the sense of Clarke for a locally Lipschitz function.

Definition 2.1 ([10]) Let $f: X \rightarrow \mathbb{R}$ be a locally Lipschitz function. The generalized directional derivative of $f$ at $x \in X$ in the direction $v \in X$, denoted by $f^{0}(x ; v)$, is defined by

$$
f^{0}(x ; v)=\limsup _{y \rightarrow x, \lambda \downarrow 0} \frac{f(y+\lambda v)-f(y)}{\lambda} .
$$

The generalized gradient or subdifferential of $f$ at $x$, denoted by $\partial f(x)$, is a subset of the dual space $X^{*}$ given by

$$
\partial f(x)=\left\{\zeta \in X^{*} \mid f^{0}(x ; v) \geq\langle\zeta, v\rangle_{X^{*} \times X} \forall v \in X\right\} .
$$

A locally Lipschitz function $f$ is said to be regular (in the sense of Clarke) at $x \in X$ if for all $v \in X$, the one-sided directional derivative $f^{\prime}(x ; v)$ exists and $f^{0}(x ; v)=f^{\prime}(x ; v)$.

We then recall the definition of pseudomonotonicity of a single-valued operator. 
Definition 2.2 ([48]) A single-valued operator $F: X \rightarrow X^{*}$ is said to be pseudomonotone, if

(i) $F$ is bounded (i.e., it maps bounded subsets of $X$ into bounded subsets of $X^{*}$ );

(ii) $u_{n} \rightarrow u$ in $X$ and $\lim \sup _{n \rightarrow \infty}\left\langle F u_{n}, u_{n}-u\right\rangle_{X^{*} \times X} \leq 0$ imply

$$
\langle F u, u-v\rangle_{X^{*} \times X} \leq \liminf _{n \rightarrow \infty}\left\langle F u_{n}, u_{n}-v\right\rangle_{X^{*} \times X} \quad \forall v \in X
$$

It can be proved (see [35], for example) that an operator $F: X \rightarrow X^{*}$ is pseudomonotone iff it is bounded and $u_{n} \rightarrow u$ in $X$ together with $\limsup _{n \rightarrow \infty}\left\langle F u_{n}, u_{n}-u\right\rangle_{X^{*} \times X} \leq 0$ imply $F u_{n} \rightarrow F u$ in $X^{*}$ and $\lim _{n \rightarrow \infty}\left\langle F u_{n}, u_{n}-u\right\rangle_{X^{*} \times X}=0$.

The following definition can be found, for example, in [38].

Definition 2.3 Let $X$ be a reflexive Banach space. A multi-valued operator $F: X \rightarrow 2^{X^{*}}$ is pseudomonotone if the following conditions hold:

(a) F has values which are nonempty, bounded, closed and convex;

(b) $F$ is upper semicontinuous from each finite dimensional subspace of $X$ into $X_{w}^{*}$;

(c) for any sequences $\left\{u_{n}\right\} \subset X$ and $\left\{u_{n}^{*}\right\} \subset X^{*}$ such that $u_{n} \rightarrow u$ in $X, u_{n}^{*} \in F u_{n}$ and $\limsup _{n \rightarrow \infty}\left\langle u_{n}^{*}, u_{n}-u\right\rangle_{X^{*} \times X} \leq 0$, we have that for every $v \in X$, there exists $u^{*}(v) \in F u$ such that

$$
\left\langle u^{*}(v), u-v\right\rangle_{X^{*} \times X} \leq \liminf _{n \rightarrow \infty}\left\langle u_{n}^{*}(v), u-v\right\rangle_{X^{*} \times X}
$$

The following proposition is usually used to check the pseudomonotonicity of an operator.

Proposition 2.4 ([12]) Let $X$ be a real reflexive Banach space, and assume that $F: X \rightarrow 2^{X^{*}}$ satisfies the following conditions:

(i) for each $v \in X, F(v)$ is a nonempty, closed and convex subset of $X^{*}$;

(ii) $F$ is bounded;

(iii) if $v_{n} \rightarrow v$ in $X, v_{n}^{*} \rightarrow v^{*}$ in $X^{*}$ with $v_{n}^{*} \in F\left(v_{n}\right)$, and $\lim _{\sup _{n \rightarrow \infty}}\left\langle v_{n}^{*}, v_{n}-v\right\rangle_{X^{*} \times X} \leq 0$, then $v^{*} \in F(v)$ and $\left\langle v_{n}^{*}, v_{n}\right\rangle_{X^{*} \times X} \rightarrow\left\langle v^{*}, v\right\rangle_{X^{*} \times X}$.

Then the operator $F$ is pseudomonotone.

We will need the notion of coercivity.

Definition 2.5 An operator $F: X \rightarrow 2^{X^{*}}$ is coercive if either $D(F)$ is bounded or $D(F)$ is unbounded and

$$
\lim _{\|u\|_{X} \rightarrow \infty, u \in D(F)} \frac{\inf \left\{\left\langle u^{*}, u\right\rangle_{X^{*} \times X} \mid u^{*} \in F u\right\}}{\|u\|_{X}}=+\infty .
$$

The following is the main surjectivity result for pseudomonotone and coercive operators. 
Theorem 2.6 ([12]) Let $X$ be a reflexive Banach space and $F: X \rightarrow 2^{X^{*}}$ be pseudomonotone and coercive. Then $F$ is surjective, i.e., $R(F)=X^{*}$.

For a Banach space $X$ and a finite time interval $I=(0, T)$, we will use the spaces $L^{p}(I ; X)$, $1 \leq p \leq \infty$. Denote by $B V(I ; X)$ the space of functions of bounded total variation on $I$ defined as follows. Let $\pi$ denote a finite partition of $\bar{I}: 0=a_{0}<a_{1}<\cdots<a_{n}=T$, and let $\mathcal{F}$ be the family of all such partitions. Then we define the total variation of a function $x: \bar{I} \rightarrow X$ as

$$
\|x\|_{B V(I ; X)}=\sup _{\pi \in \mathcal{F}} \sum_{i=1}^{n}\left\|x\left(a_{i}\right)-x\left(a_{i-1}\right)\right\|_{X} .
$$

In general, for $1 \leq q<\infty$, we similarly define

$$
\|x\|_{B V^{q}(I ; X)}^{q}=\sup _{\pi \in \mathcal{F}} \sum_{i=1}^{n}\left\|x\left(a_{i}\right)-x\left(a_{i-1}\right)\right\|_{X}^{q} .
$$

Then the space $B V^{q}(I ; X)$ consists of all the functions $x: I \rightarrow X$ such that $\|x\|_{B V^{q}(I ; X)}<\infty$.

Now for Banach spaces $X, Z$ such that $X \subset Z$ we introduce a vector space

$$
M^{p, q}(I ; X, Z)=L^{p}(I ; X) \cap B V^{q}(I ; Z) .
$$

It is a Banach space for $1 \leq p, q<\infty$ with the norm given by $\|\cdot\|_{L^{p}(I ; X)}+\|\cdot\|_{B V^{q}(I ; Z)}$.

The following result is crucial in proving convergence of the Rothe method (cf. Theorem 4.5).

Theorem 2.7 ([27]) Let $1 \leq p, q<\infty$. Let $X_{1} \subset X_{2} \subset X_{3}$ be real Banach spaces such that $X_{1}$ is reflexive, the embedding $X_{1} \subset X_{2}$ is compact and the embedding $X_{2} \subset X_{3}$ is continuous. If $\mathcal{G}$ is a bounded subset $M^{p, q}\left(I ; X_{1}, X_{3}\right)$, then it is relatively compact in $L^{p}\left(I ; X_{2}\right)$.

The following version of Aubin-Cellina convergence theorem will be used.

Theorem 2.8 ([2]) Let $X$ and $Y$ be Banach spaces. Assume $F: X \rightarrow 2^{Y}$ is a multi-valued function such that

(a) the values of $F$ are nonempty, closed and convex subsets of $Y$;

(b) $F$ is upper semicontinuous from $X$ into $Y_{w}$.

Let $x_{n}:(0, T) \rightarrow X, y_{n}:(0, T) \rightarrow Y, n \in \mathbb{N}$, be measurable functions such that $x_{n}$ converges almost everywhere on $(0, T)$ to a function $x:(0, T) \rightarrow X$ and $y_{n}$ converges weakly in $L^{1}(0, T ; Y)$ to $y:(0, T) \rightarrow Y$. If $y_{n}(t) \in F\left(x_{n}(t)\right)$ for all $n \in \mathbb{N}$ and almost all $t \in(0, T)$, then $y(t) \in F(x(t))$ for a.e. $t \in(0, T)$. 


\section{An abstract hemivariational inequality}

Let $V$ be a reflexive separable Banach space and $H$ a Hilbert space. We identify the dual space of $H$ with $H$ itself: $H^{*}=H$. We denote by $\langle\cdot, \cdot\rangle$ the duality of $V$ and $V^{*}$, and by $(\cdot, \cdot)$ the scalar product in $H$. The norms in $V$ and $H$ are $\|\cdot\|_{V}$ and $\|\cdot\|_{H}$. We denote by $|\cdot|$ the norm in $\mathbb{R}^{d}$. We consider an evolution tripe $V \subset H \subset V^{*}$ with dense, continuous and compact embeddings. Denote by $\iota: V \rightarrow H$ the embedding injection. We also introduce a reflexive Banach space $U$ and a linear, continuous opeartor $\ell: V \rightarrow U$. By $\|\iota\|$ and $\|\ell\|$ we always mean $\|\iota\|_{\mathcal{L}(V, H)}$ and $\|\ell\|_{\mathcal{L}(V, U)}$, respectively. For $T>0$, we define the spaces $\mathcal{V}=L^{2}(0, T ; V), \mathcal{H}=L^{2}(0, T ; H), \mathcal{U}=L^{2}(0, T ; U)$, $\mathcal{V}^{*}=L^{2}\left(0, T ; V^{*}\right)$ and $\mathcal{W}=\left\{v \in \mathcal{V} \mid v^{\prime} \in \mathcal{V}^{*}\right\}$, where $v^{\prime}$ is the time derivative of $v$, understood in the sense of distributions. The space $\mathcal{W}$ is embedded continuously in $C(0, T ; H)$, the space of all continuous functions $v:[0, T] \rightarrow H$ with the norm

$$
\|v\|_{C(0, T ; H)}=\max _{t \in[0, T]}\|v(t)\|_{H}
$$

We introduce the notion of a generalized Navier-Stokes type operator.

Definition 3.1 An operator $N: V \rightarrow V^{*}$ is called a generalized Navier-Stokes type operator if $N v=A v+B[v]$, where

$H(A): A \in \mathcal{L}\left(V ; V^{*}\right)$ is symmetric and for some constants $\alpha>0$ and $\beta \geq 0$,

$$
\langle A v, v\rangle \geq \alpha\|v\|_{V}^{2}-\beta\|v\|_{H}^{2} \quad \forall v \in V
$$

$H(B): B[v]:=B(v, v), B: V \times V \rightarrow V^{*}$ is a bilinear continuous operator satisfying the conditions $\langle B(u, v), v\rangle=0 \forall u, v \in V$, and $B[\cdot]: V \rightarrow V^{*}$ is weakly continuous.

Remark 3.2 If we replace the condition (3.1) by the $V$-ellipticity

$$
\langle A v, v\rangle \geq \alpha\|v\|_{V}^{2} \quad \forall v \in V
$$

then the operator $N$ of Definition 3.1 is called a Navier-Stokes type operator ([35]).

For the generalized Navier-Stokes operator $N$, obviously we have the inequality

$$
\langle N v, v\rangle \geq \alpha\|v\|_{V}^{2}-\beta\|v\|_{H}^{2} \quad \forall v \in V
$$

From [35, Lemma 9], we have the following result.

Lemma 3.3 A generalized Navier-Stokes type operator is pseudomonotone.

We consider the following inclusion: 
Problem 3.4 Find $u \in \mathcal{W}$ such that

$$
\left\{\begin{array}{l}
u^{\prime}(t)+A u(t)+B[u(t)]+\ell^{*} \partial \psi(\ell u(t)) \ni f(t) \quad \text { a.e. } t \in(0, T) \\
u(0)=u_{0} .
\end{array}\right.
$$

where $f \in \mathcal{V}^{*}, \psi: U \rightarrow \mathbb{R}, \partial \psi$ is the subdifferential of $\psi(\cdot)$ in the sense of Clarke and $\ell^{*}: U^{*} \rightarrow V^{*}$ is the adjoint operator to $\ell$.

Problem 3.4 has the following equivalent formulation.

Problem 3.5 Find $(u, \eta) \in \mathcal{W} \times \mathcal{U}^{*}$ such that

$$
\left\{\begin{array}{l}
u^{\prime}(t)+A u(t)+B[u(t)]+\ell^{*} \eta(t)=f(t) \quad \text { a.e. } t \in(0, T) \\
\eta(t) \in \partial \psi(\ell u(t)) \quad \text { a.e. } t \in(0, T) \\
u(0)=u_{0}
\end{array}\right.
$$

For our analysis, we will refer to the following conditions.

$H(\psi)$ : The functional $\psi: U \rightarrow \mathbb{R}$ satisfies

(i) $\psi$ is locally Lipschitz;

(ii) $\|\eta\|_{U^{*}} \leq c_{\psi}\left(1+\|\xi\|_{U}\right) \forall \eta \in \partial \psi(\xi), \xi \in U$, with a constant $c_{\psi}>0$;

(iii) $\left\langle\eta_{1}-\eta_{2}, \xi_{1}-\xi_{2}\right\rangle_{U^{*} \times U} \geq-m_{1}\left\|\xi_{1}-\xi_{2}\right\|_{U}^{2} \forall \eta_{i} \in \partial \psi\left(\xi_{i}\right), \xi_{i} \in U, i=1,2$, with $m_{1} \geq 0$.

$H(\ell)$ : the operator $\ell \in \mathcal{L}(V ; U)$ is compact and its Nemytskii operator $\bar{\ell}: M^{2,2}\left(0, T ; V, V^{*}\right) \rightarrow \mathcal{U}$ by $(\bar{\ell} v)(t)=\ell v(t)$ is compact.

$H(B)_{1}$ : there exists a constant $K_{1}>0$ such that

$$
\|B[v]\|{\mathcal{\mathcal { V } ^ { * }}} \leq K_{1}\|v\|_{\mathcal{V}}\|v\|_{L^{\infty}(0, T ; H)} \quad \forall v \in \mathcal{V} \cap L^{\infty}(0, T ; H) .
$$

$H(B)_{2}$ : there exist constants $K_{2}>0, \theta, \rho \in\left[0, \frac{1}{2}\right]$ such that

$$
|\langle B(u, v), u\rangle| \leq K_{2}\|u\|_{V}^{1+\theta}\|u\|_{H}^{1-\theta}\|v\|_{V}^{\rho}\|v\|_{H}^{1-\rho} \quad \forall u, v \in V .
$$

\section{Existence}

In this section we explore the solution existence. We start with a temporal semi-discrete approximation of Problem 3.5 based on the backward Euler difference, known as the Rothe method. For a fixed $N \in \mathbb{N}$, define the time step-size $k=T / N$. Introduce the piecewise constant interpolant of $f$ by

$$
f_{k, i}=\frac{1}{k} \int_{(i-1) k}^{i k} f(t) d t, \quad i=1, \ldots, N
$$


We approximate the initial condition by elements of $V$. Namely, let $\left\{u_{k, 0}\right\} \subset V$ be sequences such that $u_{k, 0} \rightarrow u_{0}$ strongly in $H$ and $\left\|u_{k, 0}\right\|_{V} \leq C / \sqrt{k}$ for some constant $C>0$. Since $V$ is dense in $H$, such a sequence $\left\{u_{k, 0}\right\}$ exists (cf. [43, Theorem 8.9]).

We will study the following Rothe scheme for the approximation of Problem 3.5.

Problem 4.1 Find $\left\{u_{k, i}\right\}_{i=0}^{N} \subset V$, and $\left\{\eta_{k, i}\right\}_{i=0}^{N} \subset U^{*}$ such that for $i=1, \ldots, N$,

$$
\left\{\begin{array}{l}
\frac{1}{k}\left(u_{k, i}-u_{k, i-1}, v\right)+\left\langle A u_{k, i}, v\right\rangle+\left\langle B\left[u_{k, i}\right], v\right\rangle+\left\langle\eta_{k, i}, \ell v\right\rangle_{U^{*} \times U}=\left\langle f_{k, i}, v\right\rangle \quad \forall v \in V, \\
\eta_{k, i} \in \partial \psi\left(\ell u_{k, i}\right) .
\end{array}\right.
$$

First we show an existence result for Problem 4.1.

Theorem 4.2 Assume $H(A), H(B), H(\psi), u_{0} \in H$ and $\alpha>c_{\psi}\|\ell\|^{2}$. Then for $k>0$ small enough, there exists a solution of Problem 4.1.

Proof. It is sufficient to prove that for a given $u_{k, i-1} \in V$, there exist $u_{k, i} \in V$ and $\eta_{k, i} \in U^{*}$ satisfying (4.1). Define a multivalued operator $L: V \rightarrow 2^{V^{*}}$ by

$$
L v=\frac{\iota^{*} \iota}{k} v+A v+B[v]+\ell^{*} \partial \psi(\ell v), \quad v \in V .
$$

Then, (4.1) is equivalent to

$$
L u_{k, i} \ni f_{k, i}+\frac{\iota^{*} \iota}{k} u_{k, i-1} .
$$

So it suffices to prove the surjectivity of $L$. By Theorem 2.6, we only need to show that $L$ is pseudomonotone and coercive.

First, we prove the coercivity of $L$. Let $v \in V$ and $v^{*} \in L v$. Then

$$
v^{*}=\frac{\iota^{*} \iota}{k} v+A v+B[v]+\ell^{*} \eta
$$

where $\eta \in \partial \psi(\ell v)$. Using $H(A)$ and $H(B)$, we have

$$
\begin{aligned}
\left\langle v^{*}, v\right\rangle & =\left(\frac{\iota^{*} \iota}{k} v, v\right)+\langle A v, v\rangle+\langle B[v], v\rangle+\langle\eta, \ell v\rangle_{U^{*} \times U} \\
& \geq\left(\frac{1}{k}-\beta\right)\|v\|_{H}^{2}+\alpha\|v\|_{V}^{2}+\langle\eta, \ell v\rangle_{U^{*} \times U} .
\end{aligned}
$$

From $H(\psi)($ ii), we have

$$
\begin{aligned}
\langle\eta, \ell v\rangle_{U^{*} \times U} & \geq-\|\ell\|\|\eta\|_{U^{*}}\|v\|_{V} \\
& \geq-c_{\psi}\|\ell\|\|v\|_{V}\left(1+\|\ell v\|_{U}\right) \\
& \geq-c_{\psi}\|\ell\|\|v\|_{V}\left(1+\|\ell\|\|v\|_{V}\right) \\
& =-c_{\psi}\|\ell\|^{2}\|v\|_{V}^{2}-c_{\psi}\|\ell\|\|v\|_{V} .
\end{aligned}
$$


It follows from (4.2) and (4.3) that

$$
\left\langle v^{*}, v\right\rangle \geq\left(\frac{1}{k}-\beta\right)\|v\|_{H}^{2}+\left(\alpha-c_{\psi}\|\ell\|^{2}\right)\|v\|_{V}^{2}-c_{\psi}\|\ell\|\|v\|_{V} .
$$

Therefore, for $k<1 / \beta$ small enough, the operator $L$ is coercive.

Next we prove that $L$ is pseudomonotone. In view of Proposition 2.4, we deduce that the operator $\iota^{*} \iota / k$ is pseudomonotone from $V$ to $V^{*}$. Since the operator $\ell: V \rightarrow U$ is compact, from [27, Lemma 2] we see that $\ell^{*} \partial \psi(\ell \cdot)$ is pseudomonotone. From Lemma 3.3, we know that $N(\cdot):=A(\cdot)+B[\cdot]$ is pseudomonotone. Since the sum of two pseudomonotone operators remains pseudomonotone (cf. [12, Proposition 1.3.68]), $L$ is pseudomonotone.

Let us establish a priori bound on the solutions of Rothe problem.

Lemma 4.3 If the assumptions in Theorem 4.2 hold, then there is a constant $M_{1}>0$, independent of $k$, such that for $k>0$ small enough,

$$
\max _{1 \leq i \leq N}\left\|u_{k, i}\right\|_{H}+\sum_{i=1}^{N}\left\|u_{k, i}-u_{k, i-1}\right\|_{H}^{2}+k \sum_{i=1}^{N}\left\|u_{k, i}\right\|_{V}^{2} \leq M_{1} .
$$

Proof. Take $v=u_{k, i}$ in (4.1),

$$
\frac{1}{k}\left(u_{k, i}-u_{k, i-1}, u_{k, i}\right)+\left\langle A u_{k, i}, u_{k, i}\right\rangle+\left\langle B\left[u_{k, i}\right], u_{k, i}\right\rangle+\left\langle\eta_{k, i}, \ell u_{k, i}\right\rangle_{U^{*} \times U}=\left\langle f_{k, i}, u_{k, i}\right\rangle .
$$

Note that

$$
\left(u_{k, i}-u_{k, i-1}, u_{k, i}\right)=\frac{1}{2}\left\|u_{k, i}\right\|_{H}^{2}-\frac{1}{2}\left\|u_{k, i-1}\right\|_{H}^{2}+\frac{1}{2}\left\|u_{k, i}-u_{k, i-1}\right\|_{H}^{2} .
$$

From $H(A)$ and $H(B)$, we obtain

$$
\left\langle A u_{k, i}, u_{k, i}\right\rangle+\left\langle B\left[u_{k, i}\right], u_{k, i}\right\rangle \geq \alpha\left\|u_{k, i}\right\|_{V}^{2}-\beta\left\|u_{k, i}\right\|_{H}^{2} .
$$

Moreover, for any $\varepsilon>0$, we have

$$
\left\langle f_{k, i}, u_{k, i}\right\rangle \leq\left\|f_{k, i}\right\|_{V^{*}}\left\|u_{k, i}\right\|_{V} \leq \frac{\varepsilon}{2}\left\|u_{k, i}\right\|_{V}^{2}+\frac{1}{2 \varepsilon}\left\|f_{k, i}\right\|_{V^{*}}^{2} .
$$

By (4.3),

$$
\left\langle\eta_{k, i}, \ell u_{k, i}\right\rangle_{U^{*} \times U} \geq-c_{\psi}\|\ell\|^{2}\left\|u_{k, i}\right\|_{V}^{2}-c_{\psi}\|\ell\|\left\|u_{k, i}\right\|_{V} .
$$

Therefore, for any $\varepsilon>0$,

$$
\begin{aligned}
\left\langle\eta_{k, i}, \ell u_{k, i}\right\rangle_{U^{*} \times U} & \geq-c_{\psi}\|\ell\|^{2}\left\|u_{k, i}\right\|_{V}^{2}-\frac{\varepsilon}{2}\left\|u_{k, i}\right\|_{V}^{2}-\frac{c_{\psi}^{2}\|\ell\|^{2}}{2 \varepsilon} \\
& =\left(-c_{\psi}\|\ell\|^{2}-\frac{\varepsilon}{2}\right)\left\|u_{k, i}\right\|_{V}^{2}-\frac{c_{\psi}^{2}\|\ell\|^{2}}{2 \varepsilon} .
\end{aligned}
$$


Thus, from (4.5), we have

$$
\left\|u_{k, i}\right\|_{H}^{2}-\left\|u_{k, i-1}\right\|_{H}^{2}+\left\|u_{k, i}-u_{k, i-1}\right\|_{H}^{2}+c_{1} k\left\|u_{k, i}\right\|_{V}^{2} \leq \frac{k}{\varepsilon}\left\|f_{k, i}\right\|_{V^{*}}^{2}+2 k \beta\left\|u_{k, i}\right\|_{H}^{2}+c_{2} k,
$$

where $c_{1}=2\left(\alpha-\varepsilon-c_{\psi}\|\ell\|^{2}\right), c_{2}=c_{\psi}^{2}\|\ell\|^{2} / \varepsilon$, and $\varepsilon>0$ is chosen so that $c_{1}>0$, e.g., $\varepsilon=$ $\left(\alpha-c_{\psi}\|\ell\|^{2}\right) / 2$.

For $1 \leq n \leq N$, we sum the inequality (4.8) for $i=1, \ldots, n$ to obtain

$$
\begin{aligned}
& \left\|u_{k, n}\right\|_{H}^{2}+\sum_{i=1}^{n}\left\|u_{k, i}-u_{k, i-1}\right\|_{H}^{2}+c_{1} k \sum_{i=1}^{n}\left\|u_{k, i}\right\|_{V}^{2} \\
& \leq\left\|u_{k, 0}\right\|_{H}^{2}+2 k \beta \sum_{i=1}^{n}\left\|u_{k, i}\right\|_{H}^{2}+\frac{1}{\varepsilon} k \sum_{i=1}^{n}\left\|f_{k, i}\right\|_{V^{*}}^{2}+c_{2} T \\
& \leq\left\|u_{k, 0}\right\|_{H}^{2}+2 k \beta \sum_{i=1}^{n}\left\|u_{k, i}\right\|_{H}^{2}+\frac{1}{\varepsilon}\|f\|_{\mathcal{V}^{*}}^{2}+c_{2} T .
\end{aligned}
$$

Taking $k<1 / 2 \beta$ and applying a discrete Gronwall lemma (cf. [22, Proposition 5.1]), from (4.9) we obtain the bound (4.4). This completes the proof.

We now construct sequences of time dependent piecewise constant and piecewise linear functions built on the solution of Rothe problem, and show the convergence of a subsequence to a solution of Problem 3.5. Define piecewise linear and piecewise constant interpolants $u_{k} \in C([0, T] ; V)$ and $\bar{u}_{k} \in L^{\infty}(0, T ; V)$ by the formulae

$$
\begin{aligned}
& u_{k}(t)=u_{k, i}+\left(\frac{t}{k}-i\right)\left(u_{k, i}-u_{k, i-1}\right) \text { for } t \in((i-1) k, i k], 1 \leq i \leq N, \\
& \bar{u}_{k}(t)=\left\{\begin{array}{l}
u_{k, i}, t \in((i-1) k, i k], 1 \leq i \leq N \\
u_{k, 0}, t=0
\end{array}\right.
\end{aligned}
$$

The piecewise constant function $\bar{\eta}_{k}:(0, T] \rightarrow U^{*}$ is given by

$$
\bar{\eta}_{k}(t)=\eta_{k, i} \quad \text { for } t \in((i-1) k, i k], 1 \leq i \leq N .
$$

Moreover, we define $f_{k}:(0, T] \rightarrow V^{*}$ as follows

$$
f_{k}(t)=f_{k, i} \quad \text { for } t \in((i-1) k, i k], 1 \leq i \leq N .
$$

We have $f_{k} \rightarrow f$ in $\mathcal{V}^{*}$ as $k \rightarrow 0$ ([8, Lemma 3.3]). Observe that the distributional derivative of $u_{k}$ is given by $u_{k}^{\prime}(t)=\left(u_{k, i}-u_{k, i-1}\right) / k$ for $t \in((i-1) k, i k), 1 \leq i \leq N$. Thus, (4.1) can be rewritten as

$$
\left\{\begin{array}{c}
\left(u_{k}^{\prime}(t), v\right)+\left\langle A \bar{u}_{k}(t), v\right\rangle+\left\langle B\left[\bar{u}_{k}(t)\right], v\right\rangle+\left\langle\bar{\eta}_{k}(t), \ell v\right\rangle_{U^{*} \times U} \\
=\left\langle f_{k}(t), v\right\rangle \quad \forall v \in V, \text { a.e. } t \in(0, T) \\
\bar{\eta}_{k}(t) \in \partial \psi\left(\ell \bar{u}_{k}(t)\right), \text { a.e. } t \in(0, T) .
\end{array}\right.
$$


We define the Nemytskii operators $\mathcal{A}, \mathcal{B}: \mathcal{V} \rightarrow \mathcal{V}^{*}$ by $(\mathcal{A} v)(t)=A(v(t)),(\mathcal{B} v)(t)=B[v(t)]$ for $v \in \mathcal{V}$ and $\bar{\ell}: \mathcal{V} \rightarrow \mathcal{U}$ by $(\bar{\ell} v)(t)=\ell v(t)$ for $v \in \mathcal{V}$ and observe that the above problem (4.10) is equivalent to

$$
\left\{\begin{array}{c}
\left(u_{k}^{\prime}, v\right)_{\mathcal{H}}+\left\langle\mathcal{A} \bar{u}_{k}, v\right\rangle_{\mathcal{V}^{*} \times \mathcal{V}}+\left\langle\mathcal{B} \bar{u}_{k}, v\right\rangle_{\mathcal{V}^{*} \times \mathcal{V}}+\left\langle\bar{\eta}_{k}, \bar{\ell} v\right\rangle_{\mathcal{U}^{*} \times \mathcal{U}} \\
=\left\langle f_{k}, v\right\rangle_{\mathcal{V}^{*} \times \mathcal{V}} \forall v \in \mathcal{V} \\
\bar{\eta}_{k}(t) \in \partial \psi\left(\left(\ell \bar{u}_{k}\right)(t)\right), \text { a.e. } t \in(0, T)
\end{array}\right.
$$

Lemma 4.4 Assume $H(A), H(B), H(B)_{1}, H(\psi), u_{0} \in H$, and $\alpha>c_{\psi}\|\ell\|^{2}$. Then there is a constant $M_{2}>0$, independent of $k$, such that for $k>0$ small enough,

$$
\left\|\bar{u}_{k}\right\|_{\mathcal{V}}+\left\|\bar{u}_{k}\right\|_{L^{\infty}(0, T ; H)}+\left\|u_{k}\right\|_{C(0, T ; H)}+\left\|u_{k}\right\|_{\mathcal{V}}+\left\|u_{k}^{\prime}\right\|_{\mathcal{V}^{*}}+\left\|\bar{\eta}_{k}\right\|_{\mathcal{U}^{*}}+\left\|\bar{u}_{k}\right\|_{M^{2,2}\left(0, T ; V, V^{*}\right)} \leq M_{2}
$$

Proof. The inequality (4.4) provides bounds on $\left\|\bar{u}_{k}\right\|_{L^{\infty}(0, T ; H)}$ and $\left\|u_{k}\right\|_{C(0, T ; H)}$ directly. Since $\left\|\bar{u}_{k}\right\|_{\mathcal{V}}^{2}=k \sum_{i=1}^{N}\left\|u_{k, i}\right\|_{V}^{2}$, the bound on $\left\|\bar{u}_{k}\right\|_{\mathcal{V}}$ also follows from (4.4). From $\left\|u_{k}\right\|_{\mathcal{V}}^{2} \leq k \sum_{i=0}^{N}\left\|u_{k, i}\right\|_{V}^{2}$, (4.4), and $\left\|u_{k, 0}\right\|_{V} \leq C / \sqrt{k}$, we get the bound on $\left\|u_{k}\right\|_{\mathcal{V}}$.

Next, using $H(\psi)($ ii) we have

$$
\begin{aligned}
\left\|\bar{\eta}_{k}\right\|_{\mathcal{U}^{*}}^{2} & =\int_{0}^{T}\left\|\bar{\eta}_{k}(t)\right\|_{U^{*}}^{2} d t \\
& \leq \int_{0}^{T}\left(2 c_{\psi}^{2}+2 c_{\psi}^{2}\|\ell\|^{2}\left\|\bar{u}_{k}(t)\right\|_{V}^{2}\right) d t \\
& =2 T c_{\psi}^{2}+2 c_{\psi}^{2}\|\ell\|^{2}\left\|\bar{u}_{k}\right\|_{\mathcal{V}}^{2} .
\end{aligned}
$$

Hence, from the bound on $\left\|\bar{u}_{k}\right\|_{\mathcal{V}}$ we get the bound on $\left\|\bar{\eta}_{k}\right\|_{\mathcal{U}^{*}}$.

From

$$
\left\langle u_{k}^{\prime}, v\right\rangle_{\mathcal{V}^{*} \times \mathcal{V}}=\left(u_{k}^{\prime}, v\right)_{\mathcal{H}}=\left\langle f_{k}, v\right\rangle_{\mathcal{V}^{*} \times \mathcal{V}}-\left\langle\mathcal{A} \bar{u}_{k}, v\right\rangle_{\mathcal{V}^{*} \times \mathcal{V}}-\left\langle\mathcal{B} \bar{u}_{k}, v\right\rangle_{\mathcal{V}^{*} \times \mathcal{V}}-\int_{0}^{T}\left\langle\bar{\eta}_{k}(t), \ell v(t)\right\rangle_{U^{*} \times U} d t
$$

we get

$$
\left\|u_{k}^{\prime}\right\|_{\mathcal{V}^{*}} \leq\left\|f_{k}\right\|_{\mathcal{V}^{*}}+\left(\int_{0}^{T}\left\|A \bar{u}_{k}(t)\right\|_{V^{*}}^{2} d t\right)^{\frac{1}{2}}+\left\|B\left[\bar{u}_{k}\right]\right\|_{\mathcal{V}^{*}}+\|\ell\|\left\|\bar{\eta}_{k}\right\|_{\mathcal{U}^{*}}
$$

Since $\bar{u}_{k} \in \mathcal{V} \cap L^{\infty}(0, T ; H)$, by $H(B)_{1}$,

$$
\left\|B\left[\bar{u}_{k}\right]\right\|_{\mathcal{V}^{*}} \leq K_{1}\left\|\bar{u}_{k}\right\|_{\mathcal{V}}\left\|\bar{u}_{k}\right\|_{L^{\infty}(0, T ; H)}
$$

Using $H(A)$, from (4.13) and (4.14), we have

$$
\left\|u_{k}^{\prime}\right\|_{\mathcal{V}^{*}} \leq\left\|f_{k}\right\|_{\mathcal{V}^{*}}+\|A\|_{\mathcal{L}\left(V, V^{*}\right)}\left\|\bar{u}_{k}\right\|_{\mathcal{V}}+K_{1}\left\|\bar{u}_{k}\right\|_{\mathcal{V}}\left\|\bar{u}_{k}\right\|_{L^{\infty}(0, T ; H)}+\|\ell\|\left\|\bar{\eta}_{k}\right\|_{\mathcal{U}^{*}}
$$

Thus, using the bounds on $\left\|\bar{u}_{k}\right\|_{\mathcal{V}},\left\|\bar{u}_{k}\right\|_{L^{\infty}(0, T ; H)}$ and $\left\|\bar{\eta}_{k}\right\|_{\mathcal{U}^{*}}$ we get the bound on $\left\|u_{k}^{\prime}\right\|_{\mathcal{V}^{*}}$. 
Finally, we bound $\left\|\bar{u}_{k}\right\|_{M^{2,2}\left(0, T ; V, V^{*}\right)}$. Let us assume that the seminorm in $B V^{2}\left(0, T ; V^{*}\right)$ of piecewise constant function $\bar{u}_{k}$ is realized by some division $0=a_{0}<a_{1}<\ldots<a_{n}=T$, and each $a_{i}$ is in different interval $\left(\left(m_{i}-1\right) k, m_{i} k\right]$, such that $\bar{u}_{k}\left(a_{i}\right)=u_{k, m_{i}}$ with $m_{0}=0, m_{n}=N$ and $m_{i+1}>m_{i}$ for $i=1, \ldots, N-1$. Thus, from the bound on $\left\|u_{k}^{\prime}\right\|_{\mathcal{V}^{*}}$, we have

$$
\begin{aligned}
\left\|\bar{u}_{k}\right\|_{B V^{2}\left(0, T ; V^{*}\right)}^{2} & =\sum_{i=1}^{n}\left\|u_{k, m_{i}}-u_{k, m_{i}-1}\right\|_{V^{*}}^{2} \\
& \leq \sum_{i=1}^{n}\left(m_{i}-m_{i-1}\right) \sum_{l=m_{i-1}+1}^{m_{i}}\left\|u_{k, l}-u_{k, l-1}\right\|_{V^{*}}^{2} \\
& \leq \sum_{i=1}^{n}\left(m_{i}-m_{i-1}\right) \sum_{l=1}^{N}\left\|u_{k, l}-u_{k, l-1}\right\|_{V^{*}}^{2} \\
& =N \sum_{l=1}^{N}\left\|u_{k, l}-u_{k, l-1}\right\|_{V^{*}}^{2}=T k \sum_{l=1}^{N}\left\|\frac{u_{k, l}-u_{k, l-1}}{k}\right\|_{V^{*}}^{2} \\
& =T \int_{0}^{T}\left\|u_{k}^{\prime}(t)\right\|_{V^{*}}^{2} d t=T\left\|u_{k}^{\prime}\right\|_{\mathcal{V}^{*}}^{2} .
\end{aligned}
$$

Consequently, from the bound on $\left\|\bar{u}_{k}\right\|_{\mathcal{V}}$, we deduce that $\bar{u}_{k}$ is bounded in $M^{2,2}\left(0, T ; V, V^{*}\right)$. This completes the proof.

Theorem 4.5 Assume $H(A), H(B), H(B)_{1}, H(\psi), H(\ell), u_{0} \in H$, and $\alpha>c_{\psi}\|\ell\|^{2}$. Let $u_{k}, \bar{u}_{k}$ be piecewise linear and piecewise constant functions built on a solution of Rothe problem 4.1. Then for $k>0$ small enough, there exists a pair $(u, \eta)$ such that for a subsequence, $u_{k} \rightarrow u$ in $\mathcal{W}$, $u_{k} \rightarrow^{*} u$ in $L^{\infty}(0, T ; H), \bar{u}_{k} \rightarrow u$ in $\mathcal{V}, \bar{u}_{k} \rightarrow^{*} u$ in $L^{\infty}(0, T ; H)$ and $\bar{\eta}_{k} \rightarrow \eta$ in $\mathcal{U}^{*}$. Moreover, the limit $(u, \eta)$ is a solution of Problem 3.5.

Proof. By the bound (4.12), we can assume that, passing to a subsequence if necessary, there exist $\bar{u} \in \mathcal{V} \cap L^{\infty}(0, T ; H), u \in \mathcal{V} \cap L^{\infty}(0, T ; H), u_{1} \in \mathcal{V}^{*}$, and $\eta \in \mathcal{U}^{*}$ such that as $k \rightarrow 0$,

$$
\begin{aligned}
& \bar{u}_{k} \rightarrow \bar{u} \text { in } \mathcal{V} \text { and } \bar{u}_{k} \rightarrow^{*} \bar{u} \text { in } L^{\infty}(0, T ; H), \\
& u_{k} \rightarrow u \text { in } \mathcal{V} \text { and } u_{k} \rightarrow^{*} u \text { in } L^{\infty}(0, T ; H), \\
& u_{k}^{\prime} \rightarrow u_{1} \text { in } \mathcal{V}^{*} \\
& \bar{\eta}_{k} \rightarrow \eta \text { in } \mathcal{U}^{*}
\end{aligned}
$$

First we show that $\bar{u}=u$. To this end, we calculate

$$
\left\|\bar{u}_{k}-u_{k}\right\|_{\mathcal{V}^{*}}^{2}=\sum_{i=1}^{N} \int_{(i-1) k}^{i k}(i k-t)^{2}\left\|\frac{u_{k, i}-u_{k, i-1}}{k}\right\|_{V^{*}}^{2} d t=\frac{k^{2}}{3}\left\|u_{k}^{\prime}\right\|_{\mathcal{V}^{*}}^{2},
$$

implying $\bar{u}_{k}-u_{k} \rightarrow 0$ in $\mathcal{V}^{*}$ as $k \rightarrow 0$. On the other hand, from (4.15) and (4.16) we have $\bar{u}_{k}-u_{k} \rightarrow \bar{u}-u$ in $\mathcal{V}$. Since the embedding $\mathcal{V} \subset \mathcal{V}^{*}$ is continuous, we also have $\bar{u}_{k}-u_{k} \rightarrow \bar{u}-u$ 
in $\mathcal{V}^{*}$. Therefore, $\bar{u}-u=0$, i.e. $\bar{u}=u$. Since $u_{k} \rightarrow u$ in $\mathcal{V}$ and $u_{k}^{\prime} \rightarrow u_{1}$ in $\mathcal{V}^{*}$, we conclude (cf. [25, Proposition 1.2]) that $u_{1}=u^{\prime}$. Thus, for all $v \in \mathcal{V}$, we obtain

$$
\left(u_{k}^{\prime}, v\right)_{\mathcal{H}}=\left\langle u_{k}^{\prime}, v\right\rangle_{\mathcal{V}^{*} \times \mathcal{V}} \rightarrow\left\langle u^{\prime}, v\right\rangle_{\mathcal{V}^{*} \times \mathcal{V}}=\left(u^{\prime}, v\right)_{\mathcal{H}}
$$

From $H(A)$, it is clear that $\mathcal{A}$ is a linear and continuous operator from $\mathcal{V}$ to $\mathcal{V}^{*}$ and is thus also weakly continuous. Since $\bar{u}_{k} \rightarrow u$ in $\mathcal{V}$, we get

$$
\left\langle\mathcal{A} \bar{u}_{k}, v\right\rangle_{\mathcal{V}^{*} \times \mathcal{V}} \rightarrow\langle\mathcal{A} u, v\rangle_{\mathcal{V}^{*} \times \mathcal{V}}
$$

From (4.12) and (4.14) we have

$$
\left\|\mathcal{B} \bar{u}_{k}\right\|_{\mathcal{V}^{*}}=\left\|B\left[\bar{u}_{k}\right]\right\|_{\mathcal{V}^{*}} \leq K_{1}\left\|\bar{u}_{k}\right\|_{\mathcal{V}}\left\|\bar{u}_{k}\right\|_{L^{\infty}(0, T ; H)}<\infty .
$$

Therefore, applying the Lebesgue-dominated convergence theorem, from $H(B)$ we get

$$
\left\langle\mathcal{B} \bar{u}_{k}, v\right\rangle_{\mathcal{V}^{*} \times \mathcal{V}}=\int_{0}^{T}\left\langle B\left[\bar{u}_{k}(t)\right], v(t)\right\rangle d t \rightarrow \int_{0}^{T}\langle B[u(t)], v(t)\rangle d t=\langle\mathcal{B} u, v\rangle_{\mathcal{V}^{*} \times \mathcal{V}}
$$

From (4.18) we get

$$
\left\langle\bar{\eta}_{k}, \bar{\ell} v\right\rangle_{\mathcal{U}^{*} \times \mathcal{U}} \rightarrow\langle\eta, \bar{\ell} v\rangle_{\mathcal{U}^{*} \times \mathcal{U}}
$$

Since $f_{k} \rightarrow f$ in $\mathcal{V}^{*}$, we have

$$
\left\langle f_{k}, v\right\rangle_{\mathcal{V}^{*} \times \mathcal{V}} \rightarrow\langle f, v\rangle_{\mathcal{V}^{*} \times \mathcal{V}}
$$

Using (4.19)-(4.23), we can pass to the limit $k \rightarrow 0$ in (4.11) and obtain

$$
\left(u^{\prime}, v\right)_{\mathcal{H}}+\langle\mathcal{A} u, v\rangle_{\mathcal{V}^{*} \times \mathcal{V}}+\langle\mathcal{B} u, v\rangle_{\mathcal{V}^{*} \times \mathcal{V}}+\langle\eta, \bar{\ell} v\rangle_{\mathcal{U}^{*} \times \mathcal{U}}=\langle f, v\rangle_{\mathcal{V}^{*} \times \mathcal{V}} \quad \forall v \in \mathcal{V}
$$

Since $\bar{u}_{k} \rightarrow u$ in $\mathcal{V}$, from $H(\ell)$ we have $\bar{\ell} \bar{u}_{k} \rightarrow \bar{\ell} u$ in $\mathcal{U}$. Thus, for a subsequence, $\bar{\ell} \bar{u}_{k}(t) \rightarrow \bar{\ell} u(t)$ in $U$ for a.e. $t \in(0, T)$. Since $\partial \psi: U \rightarrow 2^{U^{*}}$ has nonempty, closed and convex values and is upper semicontinuous from $U$ endowed with strong topology into $U^{*}$ endowed with weak topology (cf. [11, Proposition 5.6.10]), from (4.18) and Theorem 2.8 we have

$$
\eta(t) \in \partial \psi(\ell u(t)) \quad \text { a.e. } t \in(0, T) .
$$

Finally, we pass to the limit with the initial conditions on the function $u_{k}$. Since $u_{k} \rightarrow u$ in $\mathcal{V}, u_{k}^{\prime} \rightarrow u^{\prime}$ in $\mathcal{V}^{*}$, and the embedding $\mathcal{W} \subset C(0, T ; H)$ is continuous, we have $u_{k} \rightarrow u$ in $C(0, T ; H)$. The latter implies $u_{k}(t) \rightarrow u(t)$ in $H$ for all $t \in[0, T]$ (cf. [37, Lemma 4(b)]). Therefore, $u_{k, 0}=u_{k}(0) \rightarrow u(0)$ in $H$. Since by the hypothesis $u_{k, 0} \rightarrow u_{0}$ in $H$, we have $u(0)=u_{0}$. This completes the proof.

Theorem 4.5 provides a constructive approach for the solution existence of Problem 3.5. We note that the main idea is to replace time derivative with the backward difference scheme and solve the associated elliptic problem in every time step to find the solution in the consecutive points of the time mesh. Moreover, as long as one can solve the underlying elliptic problems, this method does not require any smoothing or other additional regularizing conditions. The Rothe method has been applied to study several partial differential equation models, e.g., for nonlinear partial differential equations ([43]), for variational inequalities ([39]), for hemivariational ones ([27]) and for variational-hemivariational ones ([5]). 


\section{Uniqueness and continuous dependence on data}

In this section we explore the solution uniqueness and the continuous dependence of the solution on the data $f$ and $u_{0}$ for Problem 3.5. We will need Gronwall's inequality ([1, p. 224]): Assume $f, g \in C[a, b], h \in L^{1}(a, b), h(t) \geq 0$ a.e., and

$$
f(t) \leq g(t)+\int_{a}^{t} h(s) f(s) d s, \quad t \in[a, b] .
$$

Then,

$$
f(t) \leq g(t)+\int_{a}^{t} g(s) h(s) \exp \left(\int_{s}^{t} h(r) d r\right) d s \quad \forall t \in[a, b]
$$

We further denote $\lambda>0$ to be the embedding constant of $V \subset H$ :

$$
\|\boldsymbol{v}\|_{H} \leq \lambda\|\boldsymbol{v}\|_{V} \quad \forall \boldsymbol{v} \in V
$$

We first present a result on the uniqueness of a solution to Problem 3.5.

Theorem 5.1 Assume $H(A), H(B), H(B)_{1}, H(B)_{2}, H(\psi), H(\ell), u_{0} \in H$, and $\alpha>c_{\psi}\|\ell\|^{2}+\beta \lambda^{2}$. Let $u \in \mathcal{V}$ be a solution to Problem 3.5. Then, there exists a constant $C_{0}>0$ such that

$$
\|u\|_{\mathcal{V}} \leq C_{0}
$$

If $\alpha>m_{1}\|\ell\|^{2}$, then the solution to Problem 3.5 is unique.

Proof. First we prove the bound (5.1). Since $u \in \mathcal{V}$ solves Problem 3.5, we have

$$
\left(u^{\prime}(t), u(t)\right)+\langle A u(t), u(t)\rangle+\langle B[u(t)], u(t)\rangle+\langle\eta(t), \ell u(t)\rangle_{U^{*} \times U}=\langle f(t), u(t)\rangle, \text { a.e. } t \in(0, T),
$$

where $\eta(t) \in \partial \psi(\ell u(t))$ for a.e. $t \in(0, T)$.

By (4.3),

$$
\langle\eta(t), \ell u(t)\rangle_{U^{*} \times U} \geq-c_{\psi}\|\ell\|^{2}\|u(t)\|_{V}^{2}-c_{\psi}\|\ell\|\|u(t)\|_{V} \quad \text { a.e. } t \in(0, T) .
$$

Thus, from $H(A)$ and $H(B)$ we have

$$
\frac{1}{2} \frac{d}{d t}\|u(t)\|_{H}^{2}+\left(\alpha-c_{\psi}\|\ell\|^{2}-\beta \lambda^{2}\right)\|u(t)\|_{V}^{2}-c_{\psi}\|\ell\|\|u(t)\|_{V} \leq\langle f(t), u(t)\rangle \quad \text { a.e. } t \in(0, T) .
$$

Therefore,

$$
\frac{1}{2} \frac{d}{d t}\|u(t)\|_{H}^{2}+\left(\alpha-c_{\psi}\|\ell\|^{2}-\beta \lambda^{2}\right)\|u(t)\|_{V}^{2} \leq c_{\psi}\|\ell\|\|u(t)\|_{V}+\langle f(t), u(t)\rangle \quad \text { a.e. } t \in(0, T) .
$$


Integrating (5.2) with respect to $t$ from 0 to $T$, we obtain

$$
\begin{aligned}
\frac{1}{2}\|u(T)\|_{H}^{2}-\frac{1}{2}\left\|u_{0}\right\|_{H}^{2}+\left(\alpha-c_{\psi}\|\ell\|^{2}-\beta \lambda^{2}\right)\|u\|_{\mathcal{V}}^{2} & \leq c_{\psi}\|\ell\| \int_{0}^{T}\|u(t)\|_{V} d t+\int_{0}^{T}\langle f(t), u(t)\rangle d t \\
& \leq c_{\psi} \sqrt{T}\|\ell\|\|u\|_{\mathcal{V}}+\|f\|_{\mathcal{V}^{*}}\|u\|_{\mathcal{V}} .
\end{aligned}
$$

Hence,

$$
\frac{1}{2}\|u(T)\|_{H}^{2}+\left(\alpha-c_{\psi}\|\ell\|^{2}-\beta \lambda^{2}\right)\|u\|_{\mathcal{V}}^{2} \leq\left(c_{\psi} \sqrt{T}\|\ell\|+\|f\|_{\mathcal{V}^{*}}\right)\|u\|_{\mathcal{V}}+\frac{1}{2}\left\|u_{0}\right\|_{H}^{2}
$$

Thus, (5.1) holds.

Next, let $\left(u_{1}, \eta_{1}\right),\left(u_{2}, \eta_{2}\right)$ be two solutions of Problem 3.5. Then, for a.e. $t \in(0, T)$, we have

$$
\begin{aligned}
& \left(u_{1}^{\prime}(t)-u_{2}^{\prime}(t), v\right)+\left\langle A\left(u_{1}(t)-u_{2}(t)\right), v\right\rangle+\left\langle B\left[u_{1}(t)\right]-B\left[u_{2}(t)\right], v\right\rangle \\
& \quad+\left\langle\eta_{1}(t)-\eta_{2}(t), \ell v\right\rangle_{U^{*} \times U}=0 \quad \forall v \in V .
\end{aligned}
$$

Taking $v=u_{1}(t)-u_{2}(t)$ in (5.3), we get

$$
\begin{aligned}
& \frac{1}{2} \frac{d}{d t}\left\|u_{1}(t)-u_{2}(t)\right\|_{H}^{2}+\left\langle A\left(u_{1}(t)-u_{2}(t)\right), u_{1}(t)-u_{2}(t)\right\rangle \\
& \quad+\left\langle B\left[u_{1}(t)\right]-B\left[u_{2}(t)\right], u_{1}(t)-u_{2}(t)\right\rangle \\
& \quad+\left\langle\eta_{1}(t)-\eta_{2}(t), \ell\left(u_{1}(t)-u_{2}(t)\right)\right\rangle_{U^{*} \times U}=0, \quad \text { a.e. } t \in(0, T) .
\end{aligned}
$$

By $H(\psi)($ iii), we have

$$
\begin{aligned}
\left\langle\eta_{1}(t)-\eta_{2}(t), \ell\left(u_{1}(t)-u_{2}(t)\right)\right\rangle_{U^{*} \times U} & \geq-m_{1}\left\|\ell\left(u_{1}(t)-u_{2}(t)\right)\right\|_{U}^{2} \\
& \geq-m_{1}\|\ell\|^{2}\left\|u_{1}(t)-u_{2}(t)\right\|_{V}^{2} .
\end{aligned}
$$

By $H(B)$, we have

$$
\begin{aligned}
\left\langle B\left[u_{1}(t)\right]-B\left[u_{2}(t)\right], u_{1}(t)-u_{2}(t)\right\rangle= & \left\langle B\left(u_{1}(t), u_{1}(t)\right)-B\left(u_{2}(t), u_{2}(t)\right), u_{1}(t)-u_{2}(t)\right\rangle \\
= & \left\langle B\left(u_{1}(t)-u_{2}(t), u_{1}(t)\right), u_{1}(t)-u_{2}(t)\right\rangle \\
& +\left\langle B\left(u_{2}(t), u_{1}(t)-u_{2}(t)\right), u_{1}(t)-u_{2}(t)\right\rangle \\
= & \left\langle B\left(u_{1}(t)-u_{2}(t), u_{1}(t)\right), u_{1}(t)-u_{2}(t)\right\rangle .
\end{aligned}
$$

Using $H(A)$ and $H(B)_{1}$, from (5.4)-(5.6), we obtain for a.e. $t \in(0, T)$,

$$
\begin{gathered}
\frac{1}{2} \frac{d}{d t}\left\|u_{1}(t)-u_{2}(t)\right\|_{H}^{2}+\left(\alpha-m_{1}\|\ell\|^{2}\right)\left\|u_{1}(t)-u_{2}(t)\right\|_{V}^{2}-\beta\left\|u_{1}(t)-u_{2}(t)\right\|_{H}^{2} \\
\leq K_{2}\left\|u_{1}(t)-u_{2}(t)\right\|_{V}^{1+\theta}\left\|u_{1}(t)-u_{2}(t)\right\|_{H}^{1-\theta}\left\|u_{1}(t)\right\|_{V}^{\rho}\left\|u_{1}(t)\right\|_{H}^{1-\rho} .
\end{gathered}
$$

Integrating (5.7) with respect to $t$ from 0 to $t$, we get

$$
\begin{gathered}
\left\|u_{1}(t)-u_{2}(t)\right\|_{H}^{2}+2\left(\alpha-m_{1}\|\ell\|^{2}\right) \int_{0}^{t}\left\|u_{1}(s)-u_{2}(s)\right\|_{V}^{2} d s-2 \beta \int_{0}^{t}\left\|u_{1}(s)-u_{2}(s)\right\|_{H}^{2} d s \\
\leq 2 K_{2} \int_{0}^{t}\left\|u_{1}(s)-u_{2}(s)\right\|_{V}^{1+\theta}\left\|u_{1}(s)-u_{2}(s)\right\|_{H}^{1-\theta}\left\|u_{1}(s)\right\|_{V}^{\rho}\left\|u_{1}(s)\right\|_{H}^{1-\rho} d s .
\end{gathered}
$$


Since $u_{1} \in L^{\infty}(0, T ; H)$, the right side is bounded by

$$
2 K_{2}\left\|u_{1}\right\|_{L^{\infty}(0, T ; H)} \int_{0}^{t}\left\|u_{1}(s)-u_{2}(s)\right\|_{V}^{1+\theta}\left\|u_{1}(s)-u_{2}(s)\right\|_{H}^{1-\theta}\left\|u_{1}(s)\right\|_{V}^{\rho} d s .
$$

We then bound this quantity by applying the modified Young's inequality (cf. [1, p. 45])

$$
a b \leq \frac{\delta a^{p}}{p}+\frac{\delta^{1-q} b^{q}}{q}, \quad a, b \geq 0, \delta>0,1<p, q<\infty, \frac{1}{p}+\frac{1}{q}=1
$$

with

$$
\begin{aligned}
& a=\left\|u_{1}(s)-u_{2}(s)\right\|_{V}^{1+\theta}, \quad b=\left\|u_{1}(s)-u_{2}(s)\right\|_{H}^{1-\theta}\left\|u_{1}(s)\right\|_{V}^{\rho} \\
& p=\frac{2}{1+\theta}, \quad q=\frac{2}{1-\theta}, \quad \delta=\frac{p \varepsilon}{2 K_{2} \max \left\{\left\|u_{1}\right\|_{L^{\infty}(0, T ; H)}, 1\right\}}
\end{aligned}
$$

As a result, for any $\varepsilon>0$,

$$
\begin{aligned}
& 2 K_{2} \int_{0}^{t}\left\|u_{1}(s)-u_{2}(s)\right\|_{V}^{1+\theta}\left\|u_{1}(s)-u_{2}(s)\right\|_{H}^{1-\theta}\left\|u_{1}(s)\right\|_{V}^{\rho}\left\|u_{1}(s)\right\|_{H}^{1-\rho} d s \\
& \quad \leq \varepsilon \int_{0}^{t}\left\|u_{1}(s)-u_{2}(s)\right\|_{V}^{2} d s+C(\varepsilon, \theta) \int_{0}^{t}\left\|u_{1}(s)-u_{2}(s)\right\|_{H}^{2}\left\|u_{1}(s)\right\|_{V}^{\frac{2 \rho}{1-\theta}} d s
\end{aligned}
$$

where $C(\varepsilon, \theta)>0$ depends on $\varepsilon, \theta$ and $\left\|u_{1}\right\|_{L^{\infty}(0, T ; H)}$. Taking $\varepsilon=2\left(\alpha-m_{1}\|\ell\|^{2}\right)$ and substituting (5.9) into (5.8), we obtain for $C_{1}>0$,

$$
\left\|u_{1}(t)-u_{2}(t)\right\|_{H}^{2} \leq \int_{0}^{t}\left(2 \beta+C_{1}\left\|u_{1}(s)\right\|_{V}^{\frac{2 \rho}{1-\theta}}\right)\left\|u_{1}(s)-u_{2}(s)\right\|_{H}^{2} d s .
$$

Note that $\left\|u_{1}(t)-u_{2}(t)\right\|_{H}^{2}$ is a continuous function of $t$, and $\left\|u_{1}(\cdot)\right\|_{V}^{\frac{2 \rho}{1-\theta}} \in L^{1}(0, T)$. We can apply Gronwall's inequality to (5.10) and conclude that $u_{1}=u_{2}$. This completes the proof.

Next, we establish the continuous dependence of solution of Problem 3.5 on $f$ and $u_{0}$.

Theorem 5.2 Assume that $\alpha>m\|\ell\|^{2}+\beta \lambda^{2}$, where $m=\max \left\{c_{\psi}, m_{1}\right\}$. If the assumptions $H(A)$, $H(B), H(B)_{1}, H(B)_{2}, H(\psi), H(\ell)$ hold and $u_{0} \in H$, then the mapping $\left(f, u_{0}\right) \mapsto u: \mathcal{V}^{*} \times H \rightarrow$ $C(0, T ; H)$ is Lipschitz continuous, where u denotes the unique solution to Problem 3.5.

Proof. Let $u_{1}, u_{2} \in \mathcal{W}$ be the solutions of Problem 3.5 corresponding to two right-hand sides $f_{1}, f_{2} \in \mathcal{V}^{*}$ and two initial conditions $u_{0}^{1}, u_{0}^{2} \in H$. We substract the equation satisfied by $u_{2}$ from that satisfied by $u_{1}$, and multiply the resulting equation by $v=u_{1}(t)-u_{2}(t)$ for a.e. $t \in(0, T)$. Then we have

$$
\begin{aligned}
& \left(u_{1}^{\prime}(t)-u_{2}^{\prime}(t), u_{1}(t)-u_{2}(t)\right)+\left\langle A\left(u_{1}(t)-u_{2}(t)\right), u_{1}(t)-u_{2}(t)\right\rangle \\
& \quad+\left\langle B\left[u_{1}(t)\right]-B\left[u_{2}(t)\right], u_{1}(t)-u_{2}(t)\right\rangle+\left\langle\eta_{1}(t)-\eta_{2}(t), \ell\left(u_{1}(t)-u_{2}(t)\right)\right\rangle_{U^{*} \times U} \\
& \quad=\left\langle f_{1}(t)-f_{2}(t), u_{1}(t)-u_{2}(t)\right\rangle,
\end{aligned}
$$


where $\eta_{1}(t) \in \partial \psi\left(\ell u_{1}(t)\right)$ and $\eta_{2}(t) \in \partial \psi\left(\ell u_{2}(t)\right)$ for a.e. $t \in(0, T)$. Similar to the proof of the uniqueness in Theorem 5.1, by (5.11) and Hölder's inequality, we get

$$
\begin{aligned}
\left\|u_{1}(t)-u_{2}(t)\right\|_{H}^{2} \leq & c\left(\left\|u_{0}^{1}-u_{0}^{2}\right\|_{H}^{2}+\int_{0}^{t}\left\|f_{1}(s)-f_{2}(s)\right\|_{V^{*}}^{2} d s\right) \\
& +\int_{0}^{t}\left(2 \beta+C_{2}\left\|u_{1}(s)\right\|_{V}^{\frac{2 \rho}{1-\theta}}\right)\left\|u_{1}(s)-u_{2}(s)\right\|_{H}^{2} d s,
\end{aligned}
$$

where $c>0$ depends on $\alpha, m_{1}$ and $\|\ell\|, C_{2}>0$.

Using Gronwall's inequality, from (5.12) we get

$$
\begin{aligned}
\left\|u_{1}(t)-u_{2}(t)\right\|_{H}^{2} & \leq c\left(\left\|u_{0}^{1}-u_{0}^{2}\right\|_{H}^{2}+\int_{0}^{t}\left\|f_{1}(s)-f_{2}(s)\right\|_{V^{*}}^{2} d s\right) \exp \left\{\int_{0}^{t}\left(2 \beta+C_{2}\left\|u_{1}(s)\right\|_{V}^{\frac{2 \rho}{1-\theta}}\right) d s\right\} \\
& \leq c\left(\left\|u_{0}^{1}-u_{0}^{2}\right\|_{H}^{2}+\left\|f_{1}-f_{2}\right\|_{\mathcal{V}^{*}}^{2}\right) \exp \left\{\int_{0}^{T}\left(2 \beta+C_{2}\left\|u_{1}(t)\right\|_{V}^{\frac{2 \rho}{1-\theta}}\right) d t\right\} .
\end{aligned}
$$

Note that

$$
\int_{0}^{T}\left\|u_{1}(t)\right\|_{V}^{\frac{2 \rho}{1-\theta}} d t \leq C_{3}\left(\int_{0}^{T}\left\|u_{1}(t)\right\|_{V}^{2} d t\right)^{\frac{\rho}{1-\theta}}
$$

for $\rho, \theta \in\left[0, \frac{1}{2}\right], C_{3}>0$.

Consequently, taking into account the bound (5.1), from (5.13) and (5.14) we have

$$
\begin{aligned}
\left\|u_{1}(t)-u_{2}(t)\right\|_{H}^{2} & \leq c\left(\left\|u_{0}^{1}-u_{0}^{2}\right\|_{H}^{2}+\left\|f_{1}-f_{2}\right\|_{\mathcal{V}^{*}}^{2}\right) \exp \left\{2 \beta T+C_{4}\left(\int_{0}^{T}\left\|u_{1}(t)\right\|_{V}^{2} d t\right)^{\frac{\rho}{1-\theta}}\right\} \\
& =c\left(\left\|u_{0}^{1}-u_{0}^{2}\right\|_{H}^{2}+\left\|f_{1}-f_{2}\right\|_{\mathcal{V}^{*}}^{2}\right) \exp \left\{2 \beta T+C_{4}\left\|u_{1}\right\|_{\mathcal{V}^{\frac{2 \rho}{1-\theta}}}^{1-\theta}\right\} \\
& \leq c\left(\left\|u_{0}^{1}-u_{0}^{2}\right\|_{H}^{2}+\left\|f_{1}-f_{2}\right\|_{\mathcal{V}^{*}}^{2}\right) \exp \left\{2 \beta T+C_{4} C_{0}^{\frac{2 \rho}{1-\theta}}\right\} \\
& =C_{5}\left(\left\|u_{0}^{1}-u_{0}^{2}\right\|_{H}^{2}+\left\|f_{1}-f_{2}\right\|_{\mathcal{V}^{*}}^{2}\right),
\end{aligned}
$$

where $C_{4}>0, C_{5}=c \exp \left\{2 \beta T+C_{4} C_{0}^{\frac{2 \rho}{1-\theta}}\right\}$. This completes the proof.

\section{Application to the hemivariational inequalities of the Navier-Stokes equations}

Let $\Omega$ be a bounded simply connected domain in $\mathbb{R}^{2}$ with a smooth boundary $\Gamma$. For a given $T>0$, define $Q=\Omega \times(0, T)$ and $\Sigma=\Gamma \times(0, T)$. Recall the Navier-Stokes equations for unsteady flows of incompressible viscous fluids:

$$
\begin{aligned}
& \boldsymbol{u}^{\prime}-\nu \Delta \boldsymbol{u}+(\boldsymbol{u} \cdot \nabla) \boldsymbol{u}+\nabla p=\boldsymbol{f} \quad \text { in } Q \\
& \operatorname{div} \boldsymbol{u}=0 \quad \text { in } Q
\end{aligned}
$$


where $\boldsymbol{u}(\boldsymbol{x}, t)$ is the flow velocity field, $\nu>0$ the kinematic viscosity, $p(\boldsymbol{x}, t)$ the pressure, and $\boldsymbol{f}(\boldsymbol{x}, t)$ the density of external forces. The equation (6.2) reflects the incompressibility constraint. In this section, we will apply the results on the abstract hemivariational inequality to study the boundary and domain hemivariational inequalities for the nonstationary Navier-Stokes equations (6.1)-(6.2).

\subsection{A boundary hemivariational inequality}

We rewrite the equation (6.1) in terms of the curl operator curl (see [17] for its definition and properties). Using the identities

$$
\begin{aligned}
& (\boldsymbol{u} \cdot \nabla) \boldsymbol{u}=\operatorname{curl} \boldsymbol{u} \times \boldsymbol{u}+\frac{1}{2} \nabla|\boldsymbol{u}|^{2}, \\
& -\Delta \boldsymbol{u}=\operatorname{curl} \operatorname{curl} \boldsymbol{u}-\nabla \operatorname{div} \boldsymbol{u},
\end{aligned}
$$

we can rewrite (6.1)-(6.2) as

$$
\begin{aligned}
& \boldsymbol{u}^{\prime}+\nu \operatorname{curl} \operatorname{curl} \boldsymbol{u}+\operatorname{curl} \boldsymbol{u} \times \boldsymbol{u}+\nabla h=\boldsymbol{f} \quad \text { in } Q, \\
& \operatorname{div} \boldsymbol{u}=0 \quad \text { in } Q,
\end{aligned}
$$

where $h(\boldsymbol{x}, t)=p(\boldsymbol{x}, t)+|\boldsymbol{u}(\boldsymbol{x}, t)|^{2} / 2$ is the dynamic pressure. The Navier-Stokes equations are supplemented by initial and boundary conditions. For the initial condition, we choose

$$
\boldsymbol{u}(0)=\boldsymbol{u}_{0} \quad \text { in } \Omega,
$$

where $\boldsymbol{u}_{0}$ denotes a given initial value of $\boldsymbol{u}(t)$. Here and below, $\boldsymbol{u}(t)$ stands for the function $\Omega \ni \boldsymbol{x} \mapsto \boldsymbol{u}(\boldsymbol{x}, t) \in \mathbb{R}^{2}$.

We turn to a description of the boundary condition. Let $\boldsymbol{n}=\left(n_{1}, n_{2}\right)^{\top}$ be the unit outward normal on the boundary $\Gamma$. For a vector $\boldsymbol{u}$ defined on $\Gamma$, denote by $u_{n}=\boldsymbol{u} \cdot \boldsymbol{n}$ and $\boldsymbol{u}_{\tau}=\boldsymbol{u}-u_{n} \boldsymbol{n}$ the normal and tangential component of the vector $\boldsymbol{u}$, respectively. For the boundary condition, we consider

$$
\begin{aligned}
& \boldsymbol{u}_{\tau}=\mathbf{0} \text { on } \Sigma, \\
& h(t) \in \partial j\left(t, u_{n}(t)\right) \quad \text { on } \Sigma,
\end{aligned}
$$

where $j\left(t, u_{n}(t)\right)$ is a short-hand notation for $j\left(\boldsymbol{x}, t, u_{n}(\boldsymbol{x}, t)\right) ; j: \Gamma \times(0, T) \times \mathbb{R} \rightarrow \mathbb{R}$ is called a superpotential and denotes the function which is locally Lipschitz in the third variable, $\partial j$ is the subdifferential of $j(\boldsymbol{x}, t, \cdot)$ in the sense of Clarke. The boundary condition (6.7) arises in the problem of motion of a fluid through a tube or channel: the fluid pumped into $\Omega$ can leave the tube at the boundary orifices while a device can change the sizes of the latter. In this problem we regulate the normal velocity of the fluid on the boundary to reduce the total pressure on $\Gamma$. Hence, different boundary conditions describe different physical phenomena. Note that in the case where the function $j\left(\boldsymbol{x}, t, u_{n}\right)$ is convex with respect to its last argument, the problem (6.3)-(6.7) 
leads to a variational inequality. Here, we do not assume the convexity of $j$ with respect to its last argument, and then the problem corresponds to a hemivariational inequality.

We set up a weak formulation of the problem (6.3)-(6.7). To this end, we introduce the following notation:

$$
\mathcal{M}=\left\{\boldsymbol{v} \in C^{\infty}\left(\bar{\Omega} ; \mathbb{R}^{2}\right) \mid \operatorname{div} \boldsymbol{v}=0 \text { in } \Omega, \boldsymbol{v}_{\tau}=\mathbf{0} \text { on } \Gamma\right\}
$$

Let $V$ and $H$ be the closures of $\mathcal{M}$ with respect to the norms of $H^{1}\left(\Omega ; \mathbb{R}^{2}\right)$ and $L^{2}\left(\Omega ; \mathbb{R}^{2}\right)$, respectively. Then

$$
V \subset H=H^{*} \subset V^{*}
$$

with all embeddings being dense and compact. Thus, the embedding mapping $\iota: V \rightarrow H$ is continuous and compact. Let $U=L^{2}\left(\Gamma ; \mathbb{R}^{2}\right)$ and $\ell: V \rightarrow U$. We then introduce the spaces $\mathcal{V}$, $\mathcal{H}, \mathcal{U}, \mathcal{V}^{*}$ and $\mathcal{W}$ as in Section 3. Let $\boldsymbol{f} \in \mathcal{V}^{*}$. For $\boldsymbol{v}, \boldsymbol{w}, \boldsymbol{z} \in V$, we define $A: V \rightarrow V^{*}$ and $B[\cdot]: V \rightarrow V^{*}$ by

$$
\begin{aligned}
& \langle A \boldsymbol{v}, \boldsymbol{w}\rangle=\nu \int_{\Omega} \operatorname{curl} \boldsymbol{v} \cdot \operatorname{curl} \boldsymbol{w} d x \\
& \langle B(\boldsymbol{v}, \boldsymbol{w}), \boldsymbol{z}\rangle=\int_{\Omega}(\operatorname{curl} \boldsymbol{v} \times \boldsymbol{w}) \cdot \boldsymbol{z} d x .
\end{aligned}
$$

It is known from [46] that in the case of a simply connected domain $\Omega$, the bilinear form

$$
((\boldsymbol{v}, \boldsymbol{w}))_{V}=\int_{\Omega} \operatorname{curl} \boldsymbol{v} \cdot \operatorname{curl} \boldsymbol{w} d x
$$

generates a norm in $V,\|\boldsymbol{v}\|_{V}=((\boldsymbol{v}, \boldsymbol{v}))_{V}^{1 / 2}$, which is equivalent to the $H^{1}\left(\Omega ; \mathbb{R}^{2}\right)$-norm.

Multiplying the equation of motion (6.3) by $\boldsymbol{v} \in V$ and applying the Green formula, we obtain

$$
\left\langle\boldsymbol{u}^{\prime}(t)+A \boldsymbol{u}(t)+B[\boldsymbol{u}(t)], \boldsymbol{v}\right\rangle+\int_{\Gamma} h(t) v_{n} d \Gamma=\langle\boldsymbol{f}(t), \boldsymbol{v}\rangle \quad \forall \boldsymbol{v} \in V \text {, a.e. } t \in(0, T) .
$$

From the relation (6.7), by using the definition of the Clarke subdifferential, we have

$$
\int_{\Gamma} h(t) v_{n} d \Gamma \leq \int_{\Gamma} j^{0}\left(t, u_{n}(t) ; v_{n}\right) d \Gamma
$$

where $j^{0}(t, \xi ; \zeta) \equiv j^{0}(\boldsymbol{x}, t, \xi ; \zeta)$ denotes the generalized directional derivative of $j(\boldsymbol{x}, t, \cdot)$ at the point $\xi \in \mathbb{R}$ in the direction $\zeta \in \mathbb{R}$. The last two relations together yield the following variational formulation.

Problem 6.1 Find $\boldsymbol{u} \in \mathcal{W}$ such that

$$
\left\{\begin{array}{l}
\left\langle\boldsymbol{u}^{\prime}(t)+A \boldsymbol{u}(t)+B[\boldsymbol{u}(t)], \boldsymbol{v}\right\rangle+\int_{\Gamma} j^{0}\left(t, u_{n}(t) ; v_{n}\right) d \Gamma \geq\langle\boldsymbol{f}(t), \boldsymbol{v}\rangle \quad \forall \boldsymbol{v} \in V, \text { a.e. } t \in(0, T), \\
\boldsymbol{u}(0)=\boldsymbol{u}_{0} .
\end{array}\right.
$$


Concerning the superpotential $j$, we assume the following hypothesis:

$H(j): j: \Gamma \times(0, T) \times \mathbb{R} \rightarrow \mathbb{R}$ is such that

(i) $j(\cdot, \cdot, \xi)$ is measurable on $\Sigma$ for all $\xi \in \mathbb{R}$ and there exist $e \in L^{2}(\Gamma)$ such that $j(\cdot, \cdot, e(\cdot)) \in$ $L^{1}(\Sigma)$

(ii) $j(\boldsymbol{x}, t, \cdot)$ is locally Lipschitz on $\mathbb{R}$ for a.e. $(\boldsymbol{x}, t) \in \Sigma$;

(iii) $|\zeta| \leq c_{0}(1+|\xi|)$ for all $\zeta \in \partial j(\boldsymbol{x}, t, \xi), \xi \in \mathbb{R}$, a.e. $(\boldsymbol{x}, t) \in \Sigma$ with $c_{0}>0$;

(iv) $\left(\zeta_{1}-\zeta_{2}\right)\left(\xi_{1}-\xi_{2}\right) \geq-m\left|\xi_{1}-\xi_{2}\right|^{2}$ for all $\zeta_{i} \in \partial j\left(\boldsymbol{x}, t, \xi_{i}\right), \xi_{i} \in \mathbb{R}, i=1,2$, a.e. $(\boldsymbol{x}, t) \in \Sigma$ with $m \geq 0$.

Now we consider the functional $J:(0, T) \times U \rightarrow \mathbb{R}$ defined by

$$
J(t, \boldsymbol{u})=\int_{\Gamma} j\left(\boldsymbol{x}, t, u_{n}(\boldsymbol{x})\right) d \Gamma, \quad \boldsymbol{u} \in U, \text { a.e. } t \in(0, T) .
$$

Lemma 6.2 Assume that $j: \Gamma \times(0, T) \times \mathbb{R} \rightarrow \mathbb{R}$ satisfies the hypothesis $H(j)$. Then the functional $J$ defined by (6.11) has the following properties.

(i) $J(t, \cdot)$ is locally Lipschitz on $U$ for a.e. $t \in(0, T)$;

(ii) $\|\boldsymbol{\eta}\|_{U} \leq \bar{c}\left(1+\|\boldsymbol{u}\|_{U}\right)$ for all $\boldsymbol{\eta} \in \partial J(t, \boldsymbol{u}), \boldsymbol{u} \in U$, a.e. $t \in(0, T)$ with $\bar{c}>0$, where $\bar{c}=\sqrt{2} c_{0} \max \{\sqrt{m(\Gamma)}, 1\}, m(\Gamma)$ being the measure of $\Gamma$;

(iii) $J^{0}(t, \boldsymbol{u} ; \boldsymbol{v}) \leq \int_{\Gamma} j^{0}\left(t, u_{n}(\boldsymbol{x}) ; v_{n}(\boldsymbol{x})\right) d \Gamma$ for all $\boldsymbol{u}, \boldsymbol{v} \in L^{2}\left(\Gamma ; \mathbb{R}^{2}\right)$, a.e. $t \in(0, T)$;

(iv) $\left\langle\boldsymbol{z}_{1}-\boldsymbol{z}_{2}, \boldsymbol{u}_{1}-\boldsymbol{u}_{2}\right\rangle_{U} \geq-m\left\|\boldsymbol{u}_{1}-\boldsymbol{u}_{2}\right\|_{U}^{2}$ for all $\boldsymbol{z}_{i} \in \partial J\left(t, \boldsymbol{u}_{i}\right), \boldsymbol{u}_{i} \in U, i=1,2$, a.e. $t \in(0, T)$.

Proof. We define $\tilde{j}: \Gamma \times(0, T) \times \mathbb{R}^{2} \rightarrow \mathbb{R}$ by $\widetilde{j}(\boldsymbol{x}, t, \boldsymbol{\zeta})=j\left(\boldsymbol{x}, t, \zeta_{n}\right)$ for $(\boldsymbol{x}, t, \boldsymbol{\zeta}) \in \Gamma \times(0, T) \times \mathbb{R}^{2}$. Then, $\tilde{j}(\boldsymbol{x}, t, \boldsymbol{\zeta})=j(\boldsymbol{x}, t, L \boldsymbol{\zeta})$, where $L \in \mathcal{L}\left(\mathbb{R}^{2}, \mathbb{R}\right)$ is defined by $L \boldsymbol{\zeta}=\zeta_{n}=\boldsymbol{\zeta} \cdot \boldsymbol{n}$ and that $L^{*} \in \mathcal{L}\left(\mathbb{R}, \mathbb{R}^{2}\right)$ is given by $L^{*} q=q \boldsymbol{n}$ for $q \in \mathbb{R}$ ([35, Lemma 13]). Then, in view of [38, Proposition 3.37, Theorem 3.47], we obtain the conclusions (i)-(iii). Using arguments similar to those in the proof of Theorem 4.20 in [38], we get (iv).

Now we consider the following inclusion:

Problem 6.3 Find $\boldsymbol{u} \in \mathcal{W}$ such that

$$
\left\{\begin{array}{l}
\boldsymbol{u}^{\prime}(t)+A \boldsymbol{u}(t)+B[\boldsymbol{u}(t)]+\ell^{*} \partial J(\ell \boldsymbol{u}(t)) \ni \boldsymbol{f}(t) \quad \text { a.e. } t \in(0, T), \\
\boldsymbol{u}(0)=\boldsymbol{u}_{0},
\end{array}\right.
$$

where $\partial J(\ell \boldsymbol{u}(t)) \equiv \partial J(t, \ell \boldsymbol{u}(t))$ and $\ell^{*}: U^{*} \rightarrow V^{*}$ is the adjoint operator to $\ell$.

Remark 6.4 If the functional $J$ is of the form (6.11) and $H(j)$ holds, it is clear that every solution to (6.12) is also a solution to the inequality (6.10). If either $j$ or $-j$ is regular, then the converse is also true. Indeed, from [38, Theorem 3.47(vii)] we have, for a.e. $t \in(0, T)$,

$$
\left\langle\boldsymbol{f}(t)-\boldsymbol{u}^{\prime}(t)-A \boldsymbol{u}(t)-B[\boldsymbol{u}(t)], \boldsymbol{v}\right\rangle \leq \int_{\Gamma} j^{0}\left(t, u_{n}(t) ; v_{n}\right) d \Gamma=J^{0}(t, \ell \boldsymbol{u}(t) ; \ell \boldsymbol{v}) \quad \forall \boldsymbol{v} \in V
$$


By [38, Proposition 3.37(ii)], we obtain

$$
\boldsymbol{f}(t)-\boldsymbol{u}^{\prime}(t)-A \boldsymbol{u}(t)-B[\boldsymbol{u}(t)] \in \partial(J \circ \ell)(\boldsymbol{u}(t))=\ell^{*} \partial J(\ell \boldsymbol{u}(t)), \quad \text { a.e. } t \in(0, T),
$$

which implies (6.12).

Note that the operator $N: V \rightarrow V^{*}$, defined by $N \boldsymbol{v}=A \boldsymbol{v}+B[\boldsymbol{v}]$, is a generalized Navier-Stokes type operator with $\alpha=\nu$ and $\beta=0$ ([46, Chapter II $]$ ). Thus, the assumptions $H(A)$ and $H(B)$ hold. Assume $H(j)$ and let $\psi(\cdot):=J(t, \cdot)$ for a.e. $t \in(0, T)$, and hence from Lemma 6.2 we know that $H(\psi)$ is satisfied. Observe that $\ell: V \rightarrow U$ is linear, continuous and compact. To verify the assumption $H(\ell)$, let $\left\{\boldsymbol{u}_{n}\right\}$ be a bounded sequence in $M^{2,2}\left(0, T ; V, V^{*}\right)$. Let $\delta \in\left(0, \frac{1}{2}\right)$. In view of Theorem 2.7, we deduce that the embedding $M^{2,2}\left(0, T ; V, V^{*}\right) \subset L^{2}\left(0, T ; H^{\frac{1}{2}+\delta}\left(\Omega ; \mathbb{R}^{2}\right)\right)$ is compact. Therefore, there exists a subsequence $\left\{\boldsymbol{u}_{n_{j}}\right\}$ of $\left\{\boldsymbol{u}_{n}\right\}$ such that $\boldsymbol{u}_{n_{j}} \rightarrow \boldsymbol{u}$ in $L^{2}\left(0, T ; H^{\frac{1}{2}+\delta}\left(\Omega ; \mathbb{R}^{2}\right)\right)$ for some element $\boldsymbol{u} \in L^{2}\left(0, T ; H^{\frac{1}{2}+\delta}\left(\Omega ; \mathbb{R}^{2}\right)\right)$. Since the embedding $H^{\frac{1}{2}+\delta}\left(\Omega ; \mathbb{R}^{2}\right) \subset U$ is compact, there exists a further subsequence of $\left\{\boldsymbol{u}_{n_{j}}\right\}$, still denoted by $\left\{\boldsymbol{u}_{n_{j}}\right\}$, such that $\boldsymbol{u}_{n_{j}} \rightarrow \boldsymbol{u}$ in $\mathcal{U}$. Thus, the assumption $H(\ell)$ is satisfied. The operator $B$ in (6.10) satisfies the assumption $H(B)_{1}$ with $K_{1}=\sqrt{2}$ (cf. [46, Chapter III, Lemma 3.4]). The assumption $H(B)_{2}$ is satisfied with $\theta=\rho=\frac{d}{4}=\frac{1}{2}$ by the operator $B$ in (6.10) (cf. [46, Chapter III, Section 3]).

Summarizing, for Problem 6.3, the assumptions $H(A), H(B), H(B)_{1}, H(B)_{2}$ and $H(\psi)$ are valid. Thus, applying the results from Sections 4 and 5 , we have the following statements.

Theorem 6.5 Assume $H(j), \boldsymbol{u}_{0} \in H$, and $\nu>\bar{c}\|\ell\|^{2}$, where $\bar{c}=\sqrt{2} c_{0} \max \{\sqrt{m(\Gamma)}, 1\}$. Then Problem 6.3 has a solution $(\boldsymbol{u}, \boldsymbol{\eta}) \in \mathcal{W} \times \mathcal{U}^{*}$. There exists a constant $\widetilde{C}>0$ such that $\|\boldsymbol{u}\|_{\mathcal{V}} \leq \widetilde{C}$ for any solution $\boldsymbol{u} \in \mathcal{V}$ of Problem 6.3. Moreover, if $\nu>m\|\ell\|^{2}$, then the solution to Problem 6.3 is unique and the mapping $\left(\boldsymbol{f}, \boldsymbol{u}_{0}\right) \mapsto \boldsymbol{u}$ is Lipschitz continuous from $\mathcal{V}^{*} \times H$ to $C(0, T ; H)$.

\subsection{A domain hemivariational inequality}

Here we apply the results on the abstract problem to a domain hemivariational inequality for the nonstationary Navier-Stokes equations. We begin with the following initial-boundary value problem:

$$
\begin{aligned}
& \boldsymbol{u}^{\prime}-\nu \Delta \boldsymbol{u}+(\boldsymbol{u} \cdot \nabla) \boldsymbol{u}+\nabla p=\boldsymbol{f}_{1}+\boldsymbol{f}_{2} \quad \text { in } Q \\
& \operatorname{div} \boldsymbol{u}=0 \quad \text { in } Q \\
& \boldsymbol{u}=\mathbf{0} \quad \text { on } \partial \Omega \times(0, T) \\
& \boldsymbol{u}(0)=\boldsymbol{u}_{0} \quad \text { in } \Omega
\end{aligned}
$$

where $-\boldsymbol{f}_{2}(\boldsymbol{x}, t) \in \partial \bar{j}(t, \boldsymbol{u}(t))$ in $Q, \bar{j}: \Omega \times(0, T) \times \mathbb{R}^{2} \rightarrow \mathbb{R}$ is a superpotential which is locally Lipschitz in the third variable.

Define

$$
\mathcal{N}=\left\{\boldsymbol{v} \in C_{0}^{\infty}\left(\bar{\Omega} ; \mathbb{R}^{2}\right) \mid \operatorname{div} \boldsymbol{v}=0 \text { in } \Omega\right\}
$$


Let $V$ and $H$ be the closures of $\mathcal{N}$ in $H_{0}^{1}\left(\Omega ; \mathbb{R}^{2}\right)$ and $L^{2}\left(\Omega ; \mathbb{R}^{2}\right)$, respectively. Then

$$
V \subset H=H^{*} \subset V^{*}
$$

with all embeddings being dense and compact. Let $U=L^{2}\left(\Omega ; \mathbb{R}^{2}\right)$ and $\ell: V \rightarrow U$. Again, we then introduce the spaces $\mathcal{V}, \mathcal{H}, \mathcal{U}, \mathcal{V}^{*}$ and $\mathcal{W}$ as in Section 3. Let $\boldsymbol{f}_{1} \in \mathcal{V}^{*}$. For $\boldsymbol{v}, \boldsymbol{w}, \boldsymbol{z} \in V$, we define $A: V \rightarrow V^{*}$ and $B[\cdot]: V \rightarrow V^{*}$ by

$$
\begin{aligned}
& \langle A \boldsymbol{v}, \boldsymbol{w}\rangle=\nu \int_{\Omega} \nabla \boldsymbol{v} \cdot \nabla \boldsymbol{w} d x, \\
& \langle B(\boldsymbol{v}, \boldsymbol{w}), \boldsymbol{z}\rangle=\int_{\Omega}(\boldsymbol{v} \cdot \nabla) \boldsymbol{w} \cdot \boldsymbol{z} d x .
\end{aligned}
$$

Similar to Subsection 6.1, we can derive the following weak formulation of (6.13)-(6.16):

Problem 6.6 Find $\boldsymbol{u} \in \mathcal{W}$ such that

$$
\left\{\begin{array}{l}
\left\langle\boldsymbol{u}^{\prime}(t)+A \boldsymbol{u}(t)+B[\boldsymbol{u}(t)], \boldsymbol{v}\right\rangle+\int_{\Omega} \bar{j}^{0}(t, \boldsymbol{u}(t) ; \boldsymbol{v}) d x \geq\left\langle\boldsymbol{f}_{1}(t), \boldsymbol{v}\right\rangle \quad \forall \boldsymbol{v} \in V, \text { a.e. } t \in(0, T), \\
\boldsymbol{u}(0)=\boldsymbol{u}_{0}
\end{array}\right.
$$

Define a functional $\bar{J}:(0, T) \times U \rightarrow \mathbb{R}$ by

$$
\bar{J}(t, \boldsymbol{u})=\int_{\Omega} \bar{j}(\boldsymbol{x}, t, \boldsymbol{u}(\boldsymbol{x})) d x, \quad \boldsymbol{u} \in U, \text { a.e. } t \in(0, T) .
$$

Concerning the superpotential $\bar{j}$, we assume the following hypothesis:

$H(\bar{j}): \bar{j}: \Omega \times(0, T) \times \mathbb{R}^{2} \rightarrow \mathbb{R}$ is such that

(i) $\bar{j}(\cdot, \cdot, \boldsymbol{\xi})$ is measurable on $Q$ for all $\boldsymbol{\xi} \in \mathbb{R}^{2}$ and there exists $\boldsymbol{e} \in L^{2}\left(\Omega ; \mathbb{R}^{2}\right)$ such that $\bar{j}(\cdot, \cdot, \boldsymbol{e}(\cdot)) \in L^{1}(Q)$;

(ii) $\bar{j}(\boldsymbol{x}, t, \cdot)$ is locally Lipschitz on $\mathbb{R}^{2}$ for a.e. $(\boldsymbol{x}, t) \in Q$;

(iii) $|\boldsymbol{\zeta}| \leq c_{0}(1+|\boldsymbol{\xi}|)$ for all $\boldsymbol{\zeta} \in \partial \bar{j}(\boldsymbol{x}, t, \boldsymbol{\xi}), \boldsymbol{\xi} \in \mathbb{R}^{2}$, a.e. $(\boldsymbol{x}, t) \in Q$ with $c_{0}>0$;

(iv) $\left(\boldsymbol{\zeta}_{1}-\boldsymbol{\zeta}_{2}\right) \cdot\left(\boldsymbol{\xi}_{1}-\boldsymbol{\xi}_{2}\right) \geq-m\left|\boldsymbol{\xi}_{1}-\boldsymbol{\xi}_{2}\right|^{2}$ for all $\boldsymbol{\zeta}_{i} \in \partial \bar{j}\left(\boldsymbol{x}, t, \boldsymbol{\xi}_{i}\right), \boldsymbol{\xi}_{i} \in \mathbb{R}^{2}, i=1,2$, a.e. $(\boldsymbol{x}, t) \in Q$ with $m \geq 0$.

We consider the following inclusion:

Problem 6.7 Find $\boldsymbol{u} \in \mathcal{W}$ such that

$$
\left\{\begin{array}{l}
\boldsymbol{u}^{\prime}(t)+A \boldsymbol{u}(t)+B[\boldsymbol{u}(t)]+\ell^{*} \partial \bar{J}(\ell \boldsymbol{u}(t)) \ni \boldsymbol{f}_{1}(t) \quad \text { a.e. } t \in(0, T) \\
\boldsymbol{u}(0)=\boldsymbol{u}_{0}
\end{array}\right.
$$

where $\partial \bar{J}(\ell \boldsymbol{u}(t)) \equiv \partial \bar{J}(t, \ell \boldsymbol{u}(t))$ and $\ell^{*}: U^{*} \rightarrow V^{*}$ is the adjoint operator to $\ell$. 
Observe that for Problem 6.7, the assumptions $H(A), H(B), H(B)_{1}, H(B)_{2}$ and $H(\psi)$ are satisfied. Similar to Theorem 6.5, we can get the results on the existence, uniqueness and continuous dependence of solutions of Problem 6.7.

Theorem 6.8 Assume $H(\bar{j}), \boldsymbol{u}_{0} \in H$, and $\nu>\bar{c}\|\ell\|^{2}$, where $\bar{c}=\sqrt{2} c_{0} \max \{\sqrt{m(\Omega)}, 1\}, m(\Omega)$ being the measure of $\Omega$. Then Problem 6.7 has a solution $(\boldsymbol{u}, \boldsymbol{\eta}) \in \mathcal{W} \times \mathcal{U}^{*}$. There exists a constant $\widetilde{C}>0$ such that $\|\boldsymbol{u}\|_{\mathcal{V}} \leq \widetilde{C}$ for any solution $\boldsymbol{u} \in \mathcal{V}$ of Problem 6.7. Moreover, if $\nu>m\|\ell\|^{2}$, then the solution to Problem 6.7 is unique and the mapping $\left(\boldsymbol{f}, \boldsymbol{u}_{0}\right) \mapsto \boldsymbol{u}$ is Lipschitz continuous from $\mathcal{V}^{*} \times H$ to $C(0, T ; H)$.

\section{References}

[1] K. Atkinson and W. Han, Theoretical Numerical Analysis: A Functional Analysis Framework, third edition, Springer, New York, 2009.

[2] J. P. Aubin and H. Frankowska, Set-Valued Analysis, Birkhäuser, Boston, Basel, Berlin, 1990.

[3] C. Baiocchi and A. Capelo, Variational and Quasivariational Inequalities: Applications to Free-Boundary Problems, Wiley, Chichester, 1984.

[4] M. Barboteu, K. Bartosz, W. Han, and T. Janiczko, Numerical analysis of a hyperbolic hemivariational inequality arising in dynamic contact, SIAM J. Numer. Anal. 53 (2015), $527-550$.

[5] K. Bartosz, X. Cheng, P. Kalita, Y. Yu, and C. Zheng, Rothe method for parabolic variationalhemivariational inequalities, J. Math. Anal. Appl. 423 (2015), 841-862.

[6] H. Brézis, Problèmes unilatéraux, J. Math. Pures et Appl. 51 (1972), 1-168.

[7] S. Carl, V. K. Le, and D. Motreanu, Nonsmooth Variational Problems and Their Inequalities: Comparison Principles and Applications, Springer, New York, 2007.

[8] C. Carstensen and J. Gwinner, A theory of discretisation for nonlinear evolution inequalities applied to parabolic Signorini problems, Ann. Mat. Pura Appl. 177 (1999), 363-394.

[9] F. H. Clarke, Generalized gradients and applications, Trans. Am. Math. Soc. 205 (1975), $247-262$.

[10] F. H. Clarke, Optimization and Nonsmooth Analysis, Wiley, Interscience, New York, 1983.

[11] Z. Denkowski, S. Migórski, and N. S. Papageorgiou, An Introduction to Nonlinear Analysis: Theory, Kluwer Academic, Plenum Publishers, Boston, Dordrecht, London, New York, 2003. 
[12] Z. Denkowski, S. Migórski, and N. S. Papageorgiou, An Introduction to Nonlinear Analysis: Applications, Kluwer Academic, Plenum Publishers, Boston, Dordrecht, London, New York, 2003.

[13] J. K. Djoko and M. Mbehou, Finite element analysis for Stokes and Navier-Stokes equations driven by threshold slip boundary conditions, Int. J. Numer. Anal. Model., Series B 4 (2013), $235-255$.

[14] G. Duvaut and J.-L. Lions, Inequalities in Mechanics and Physics, Springer-Verlag, Berlin, 1976.

[15] G. Fichera, Problemi elastostatici con vincoli unilaterali. II. Problema di Signorini con ambique condizioni al contorno, Mem. Accad. Naz. Lincei, S. VIII, Vol. VII, Sez. I 5 (1964), 91-140.

[16] H. Fujita, Flow Problems with Unilateral Boundary Conditions, College de France, Lecons, France, 1993.

[17] V. Girault and P. A. Raviart, Finite Element Methods for Navier-Stokes Equations: Theory and Algorithms, Springer-Verlag, Berlin, 1986.

[18] R. Glowinski, Numerical Methods for Nonlinear Variational Problems, Springer, New York, 1984 .

[19] R. Glowinski, J.-L. Lions, and R. Trémolières, Numerical Analysis of Variational Inequalities, North-Holland, Amsterdam, 1981.

[20] W. Han, S. Migórski, and M. Sofonea, A class of variational-hemivariational inequalities with applications to frictional contact problems, SIAM J. Math. Anal. 46 (2014), 3891-3912.

[21] W. Han and B.D. Reddy, Plasticity: Mathematical Theory and Numerical Analysis, Second Edition, Springer-Verlag, 2013.

[22] W. Han, M. Shillor, and M. Sofonea, Variational and numerical analysis of a quasistatic viscoelastic problem with normal compliance, friction and damage, J. Comput. Appl. Math. 137 (2001), 377-398.

[23] W. Han and M. Sofonea, Quasistatic Contact Problems in Viscoelasticity and Viscoplasticity, American Mathematical Society, Providence, RI-Intl. Press, Sommerville, MA, 2002

[24] P. Hartman and G. Stampacchia, On some nonlinear elliptic differential functional equations, Acta Math. 15 (1966), 271-310.

[25] J. Haslinger, M. Miettinen, and P. D. Panagiotopoulos, Finite Element Method for Hemivariational Inequalities: Theory, Methods and Applications, Kluwer Academic Publishers, Dordrecht, Boston, London, 1999. 
[26] I. Hlaváček, J. Haslinger, J. Nečas and J. Lovíšek, Solution of Variational Inequalities in Mechanics, Springer-Verlag, New York, 1988.

[27] P. Kalita, Convergence of Rothe scheme for hemivariational inequalities of parabolic type, Int. J. Numer. Anal. Model. 10 (2013), 445-465.

[28] N. Kikuchi and J.T. Oden, Contact Problems in Elasticity: A Study of Variational Inequalities and Finite Element Methods, SIAM, Philadelphia, 1988.

[29] D. Kinderlehrer and G. Stampacchia, An Introduction to Variational Inequalities and their Applications, Academic Press, New York, 1980.

[30] C. Le Roux and A. Tani, Steady solutions of the NavierStokes equations with threshold slip boundary conditions, Math. Meth. Appl. Sci. 30 (2007), 595-624.

[31] Y. Li and R. An, Semi-discrete stabilized finite element methods for Navier-Stokes equations with nonlinear slip boundary conditions based on regularization procedure, Numer. Math. 117 (2011), 1-36.

[32] Y. Li and K. Li, Existence of the solution to stationary Navier-Stokes equations with nonlinear slip boundary conditions, J. Math. Anal. Appl. 381 (2011), 1-9.

[33] Y. Li and K. Li, Global strong solutions of two-dimensional Navier-Stokes equations with nonlinear slip boundary conditions, J. Math. Anal. Appl. 393 (2012), 1-13.

[34] J.-L. Lions and G. Stampacchia, Variational inequalities, Commun. Pure Appl. Anal. 20 (1967), 493-519.

[35] S. Migórski and A. Ochal, Hemivariational inequalities for stationary Navier-Stokes equations, J. Math. Anal. Appl. 306 (2005), 197-217.

[36] S. Migórski and A. Ochal, Navier-Stokes problems modeled by evolution hemivariational inequalities, Discrete and Continuous Dynamical Systems (Supplement) (2007), 731-740.

[37] S. Migórski and A. Ochal, Quasi-static hemivariational inequality via vanishing acceleration approach, SIAM J. Math. Anal. 41 (2009), 1415-1435.

[38] S. Migórski, A. Ochal, and M. Sofonea, Nonlinear Inclusions and Hemivariational Inequalities. Models and Analysis of Contact Problems, Advances in Mechanics and Mathematics 26, Springer, New York, 2013.

[39] H. Nagase, On an application of Rothe method to nonlinear parabolic variational inequalities, Funkcial. Ekvac. 32 (1989), 273-299.

[40] Z. Naniewicz and P. D. Panagiotopoulos, Mathematical Theory of Hemivariational Inequalities and Applications, Dekker, New York, 1995. 
[41] P. D. Panagiotopoulos, Nonconvex energy functions, hemivariational inequalities and substationary principles, Acta Mechanica 42 (1983), 160-183.

[42] P. D. Panagiotopoulos, Hemivariational Inequalities, Applications in Mechanics and Engineering, SpringerVerlag, Berlin, 1993.

[43] T. Roubicek, Nonlinear Partial Differential Equations with Applications, Birkhäuser Verlag, Basel, Boston, Berlin, 2005.

[44] A. Signorini, Sopra a une questioni di elastostatica, Atti della Società Italiana per il Progresso delle Scienze, 1933.

[45] G. Stampacchia, Formes bilineaires coercitives sur les ensembles convexes, C. R. Acad. Sci. 258 (1964), 4413-4416.

[46] R. Temam, Navier-Stokes Equations: Theory and Numerical Analysis, North-Holland, Amsterdam, 1979.

[47] R. Temam, The Navier-Stokes Equations and Nonlinear Functional Analysis, CBMS-NSF Regional Conference Series in Applied Mathematics, SIAM, 1983.

[48] E. Zeidler, Nonlinear Functional Analysis and its Applications, Vol. II/B: Nonlinear Monotone Operators, Springer-Verlag, New York, Berlin, Heidelberg, 1990. 